\title{
ANALYSIS OF A CONTINUOUS FINITE ELEMENT METHOD FOR HYPERBOLIC EQUATIONS*
}

\author{
RICHARD S. FALK † AND GERARD R. RICHTER $\ddagger$
}

\begin{abstract}
A finite element method for hyperbolic equations is analyzed in the context of a first order linear problem in $R^{2}$. The method is applicable over a triangulation of the domain, and produces a continuous piecewise polynomial approximation, which can be developed in an explicit fashion from triangle to triangle. In a sense, it extends the basic upwind difference scheme to higher order. The method is shown to be stable, and error estimates are obtained. For $n$th degree approximation, the errors in the approximate solution and its gradient are shown to be at least of order $h^{n+1 / 4}$ and $h^{n-1 / 2}$, respectively, assuming sufficient regularity in the solution.
\end{abstract}

Key words. finite element method, hyperbolic equation

AMS(MOS) subject classifications. Primary 65M15, 65N30

1. Introduction. In this paper, we analyze a finite element method for the first order scalar hyperbolic equation

$$
\begin{aligned}
& \alpha \cdot \nabla u+\beta u=f \text { in } \Omega, \\
& u=g \text { on the inflow boundary } \Gamma_{\text {in }}(\Omega),
\end{aligned}
$$

where $\alpha$ is a unit vector and $\Omega$ is a bounded polygonal domain in $R^{2}$. The method produces a continuous piecewise polynomial approximation to $u$ over a triangulation of $\Omega$, and was first reported in the literature by Reed and Hill [8].

A contrasting and more common finite element approach to (1), applicable when the independent variables are time and space, is that of applying a finite element discretization in space only, then solving numerically the resulting system of ordinary differential equations. See, for example, [1] and [4]. Examples of hyperbolic equations of practical interest which do not involve time and are not directly amenable to this approach are the neutron transport equation [7] and the problem of determining the diffusion coefficient $a(x)$ in

$$
\nabla p \cdot \nabla a+a \Delta p=f .
$$

The latter is an inverse problem arising in flow through porous media [2], [5], [9].

Several techniques for obtaining full finite element discretizations of (1) have been reported in the literature. Reed and Hill [8] have provided computational results for the scheme that is the focal point of this paper and also for two other schemes, one of which produces a discontinuous approximation. The discontinuous method has been analyzed by Lesaint and Raviart [7], and more recently by Johnson and Pitkaranta [6], who obtained improved estimates. In a related work, Winther [11] obtained optimal order error estimates for a continuous finite element method applicable over a rectangular mesh.

To describe the method which we shall analyze, we let $\Delta_{h}$ be a quasiuniform triangulation of $\Omega$, constructed so that no triangle has a side parallel to the characteristic direction at any point. For any subdomain $\Omega_{S}$ of $\Omega$, we denote by $\Gamma_{i n}\left(\Omega_{S}\right)$ the inflow

\footnotetext{
* Received by the editors March 4, 1985; accepted for publication (in revised form) April 4, 1986.

$\dagger$ Department of Mathematics, Rutgers University, New Brunswick, New Jersey 08903. The work of this author was supported by National Science Foundation grant DMS-8402616.

$\ddagger$ Department of Computer Science, Rutgers University, New Brunswick, New Jersey 08903.
} 
portion of the boundary of $\Omega_{s}$, i.e., $\left\{x \in \Gamma\left(\Omega_{S}\right) \mid \alpha \cdot n<0\right\}$, where $n$ is the unit outward normal to $\Omega_{s}$, and by $\Gamma_{\text {out }}\left(\Omega_{s}\right)$ the remaining (outflow) portion of $\Gamma\left(\Omega_{s}\right)$. With $\Delta_{h}$ as above, each triangle has one inflow side and two outflow sides (a type I triangle) or two inflow sides and one outflow side (a type II triangle). Furthermore, the triangles $\left\{T_{i}\right\}$ in $\Delta_{h}$ may be ordered so that

$$
\Gamma_{\text {in }}\left(T_{k}\right) \subseteq \Gamma_{\text {out }}\left(\bigcup_{i<k} T_{i}\right) \cup\left(\Gamma_{\text {in }}(\Omega)-\Gamma_{\text {in }}\left(\bigcup_{i<k} T_{i}\right)\right)
$$

Equivalently, for each $k$, the domain of dependence of $T_{k}$ contains none of $T_{k+1}, T_{k+2}, \cdots$. This was shown in [7] for constant $\alpha$ and will be proved in the Appendix for smooth variable $\alpha$ (the assumption we make in our analysis). This ordering allows an approximate solution to be developed in an explicit manner, first in $T_{1}$, then in $T_{2}$, etc. At the point when the solution is to be formed in a given triangle, it will be known along the inflow to that triangle.

We seek an approximate solution in the subspace

$$
S_{h}^{n}=\left\{v_{h} \in C^{0}(\Omega) \text { such that }\left.v_{h}\right|_{T} \in \mathbf{P}_{n}(T)\right\},
$$

where $\mathbf{P}_{n}(T)$ denotes the space of polynomials of degree $\leqq n$ over the triangle $T$. Letting $g_{I}$ be a suitable interpolant of $g$ in $\left.S_{h}^{n}\right|_{\Gamma_{i n}(\Omega)}$ and denoting the $L^{2}$ inner product over $T$ by $(,)_{T}$, we describe the finite element method of interest as follows. type I

Problem $P_{h}$. Find $u_{h} \in S_{h}^{n}$ such that $u_{h}=g_{I}$ on $\Gamma_{i n}(\Omega)$, and for triangles of

$$
\left(\alpha \cdot \nabla u_{h}+\beta u_{h}, v_{h}\right)_{T}=\left(f, v_{h}\right)_{T} \text { for all } v_{h} \in \mathbf{P}_{n-1}(T),
$$

while for triangles of type II

$$
\left(\alpha \cdot \nabla u_{h}+\beta u_{h}, v_{h}\right)_{T}=\left(f, v_{h}\right)_{T} \text { for all } v_{h} \in \mathbf{P}_{n-2}(T) .
$$

Note that the approximate solution $u_{h}$ has a total of $\sigma_{n} \equiv \sum_{j=1}^{n+1} j$ degrees of freedom in each triangle. In a one-inflow-side triangle, there are $n+1$ degrees of freedom in $u_{h}$ along the inflow, leaving a total of $\sigma_{n-1}$ to be determined from (3). In a two-inflowside triangle, there are $2 n+1$ degrees of freedom in $u_{h}$ along the inflow, leaving $\sigma_{n-2}$ to be determined from (4). Thus in both (3) and (4), the number of equations equals the number of unknowns.

We shall assume in our analysis that $n \geqq 2$, although the case $n=1$ is also of some interest. The latter is a degenerate case in which (4) is vacuous and (3) completely determines the approximate solution. A simple calculation reveals that for a mesh of right triangles with $\alpha=$ constant, $\beta=0$, and $f=0, u_{h}$ at the triangle vertices is given by the upwind difference scheme. Thus the finite element method (3), (4) may be viewed as an extension of the upwind difference scheme to higher order and nonuniform meshes.

We note that other continuous finite element methods besides the one analyzed in this paper may be applied over triangles. For example, in place of low order polynomials, one might use as test functions the Lagrange basis functions that are unity at the unknown points. Numerical experiments in [8] indicate that this method does not perform as well as the one analyzed in this paper.

A natural question is how the continuous method described by (3), (4) compares with the discontinuous Galerkin method, which is fairly well established theoretically. In the case of the latter, one updates the solution on both type I and type II triangles 
$T$ as follows: Given $u_{-}^{h}$ on $\Gamma_{i n}(T)$, find $\left.u^{h}\right|_{T} \in \mathbf{P}_{n}(T)$ such that

$$
\begin{aligned}
\left(\alpha \cdot \nabla u^{h}\right. & \left.+\beta u^{h}, v^{h}\right)_{T}-\int_{\Gamma_{\text {in }}(T)} u_{+}^{h} v^{h} \alpha \cdot n \\
& =\left(f, v^{h}\right)_{T}-\int_{\Gamma_{\text {in }}(T)} u_{-}^{h} v^{h} \alpha \cdot n \text { for all } v^{h} \in \mathbf{P}_{n}(T)
\end{aligned}
$$

where $v_{-}(x)=\lim _{s \rightarrow 0-} v(x+s \alpha)$, and $v_{+}(x)=\lim _{s \rightarrow 0+} v(x+s \alpha)$, for $x$ lying on a side common to two triangles. Hence to update the solution on a triangle requires the solution of a $\sigma_{n} \times \sigma_{n}$ linear system of equations. When the case of piecewise quadratics is taken as an example, the discontinuous method yields a $6 \times 6$ system on each triangle, whereas the continuous method produces a $1 \times 1$ system on type I triangles and a $3 \times 3$ system on type II triangles. This illustrates the main advantage of the continuous method: it is less costly to apply. In terms of existing error estimates for the two schemes, using piecewise polynomials of degree $\leqq n$, the discontinuous Galerkin method has the error estimates

$$
\left\|u-u^{h}\right\|_{L_{2}(\Omega)}=O\left(h^{n+1 / 2}\right)
$$

and hence, using inverse properties of the subspace,

$$
\left\|u-u^{h}\right\|_{H^{1}(\Omega)}=O\left(h^{n-1 / 2}\right) .
$$

In this paper, we prove that the continuous finite element method satisfies the same $H^{1}$ estimate and an $L_{2}$ estimate with a power of $h$ one quarter less. All this analysis assumes sufficient regularity of the solution. In the last section of the paper, we present the results of some computational experiments, which show that on very smooth solutions, with a regular mesh, it is possible to achieve optimal order approximation. The same is true for the discontinuous Galerkin method. One important feature of the discontinuous Galerkin method is that it produces good results on problems with discontinuous solutions. We have not undertaken a detailed study of the continuous method for such problems; however, we provide computational results for a numerical experiment with discontinuous initial data.

The next section of this paper further describes our notation and the hypotheses under which our results will be established. Section 3 contains an existence and uniqueness proof for the approximate solution and a derivation of some of its local properties. Two basic identities are derived in $\S 4$ and then used in $\S 5$ to prove stability of the method. Error estimates are given in $\S 6$, followed by numerical results in $\S 7$.

2. Assumptions and notation. For $D$ a domain in $R^{2}$, let $\|f\|_{D}=\left(\int_{D} f^{2} d x d y\right)^{1 / 2}$, and for $\Gamma$ a line segment, let $|f|_{\Gamma}=\left(\int_{\Gamma} f^{2} d \tau\right)^{1 / 2}$. Let \|\|$_{n, D}$ denote the norm in the Sobolev space $H^{n}(D)$ and \|\|$_{\infty, D}$ and \|\|$_{1, \infty, D}$ the norms in the Sobolev spaces $L^{\infty}(D)$ and $\left[W^{1, \infty}(D)\right]^{2}$, respectively. We assume that $\alpha \in\left[W^{1, \infty}(D)\right]^{2}$ and $\beta \in L^{\infty}(D)$ for the coefficients in (1). Henceforth, we shall omit the subscript $D$ when $D=\Omega$. We also denote by $P_{k} f$ the $L^{2}$ projection over $T$ into $\mathbf{P}_{k}(T)$ (the space of polynomials of degree $\leqq k$ over $T$ ).

It will be convenient to have the following notation relative to an arbitrary triangle $T$ of $\Delta_{h}$. For $i=1,2,3$, we denote by $\Gamma_{i}$ the sides of $T$ numbered counterclockwise, by $a_{i}$ the vertices of $T$ opposite $\Gamma_{i}$, by $n_{i}$ the unit outward normals to $\Gamma_{i}$, and by $\tau_{i}$ the unit tangents along $\Gamma_{i}$ taken in a counterclockwise direction. We shall always take $\Gamma_{3}$ to be the inflow side of a type I triangle or the outflow side of a type II triangle. This notation is illustrated in Fig. 2.1. 


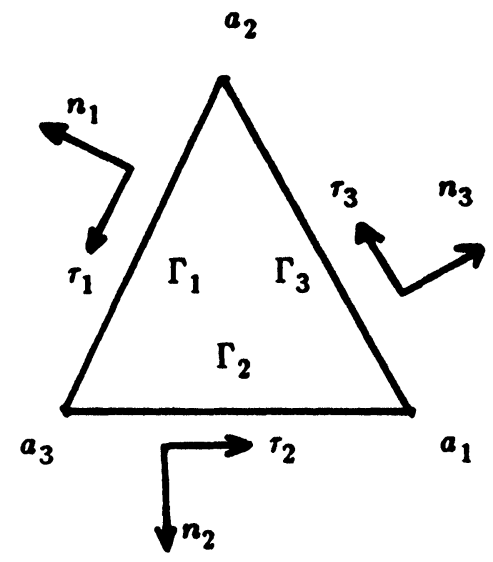

FIG. 2.1

For computational and theoretical purposes, it is advantageous to think of the triangles in a given triangulation $\Delta_{h}$ as partitioned into layers $S_{i}$. We define these as follows:

$$
\begin{aligned}
& S_{1}=\left\{T \in \Delta_{h} \mid \Gamma_{i n}(T) \subseteq \Gamma_{i n}(\Omega)\right\}, \\
& S_{i+1}=\left\{T \in \Delta_{h} \mid \Gamma_{i n}(T) \subseteq \Gamma_{i n}\left(\Omega-\bigcup_{k \leqq i} S_{k}\right)\right\}, \quad i=1,2, \cdots .
\end{aligned}
$$

With this partition of $\Delta_{h}$, the approximate solution may be obtained in an explicit manner, first in $S_{1}$, then in $S_{2}$, etc. Within each layer, the approximate solution can be obtained in parallel since the solution in any of the triangles within a layer does not depend on the solution in other triangles in that layer. This is illustrated in Fig. 2.2, with the number inside each triangle indicating the layer to which it belongs.

In our analysis, we assume that $\left\{\Delta_{h}\right\}$ is a family of triangulations of $\Omega$ satisfying the following hypotheses:

H1: All angles of all triangles are uniformly bounded away from zero.

$\mathrm{H} 2:|\alpha \cdot n|$ is uniformly bounded away from zero along all sides of all triangles. (This assumption is essential in our analysis but does not appear to be necessary computationally.)

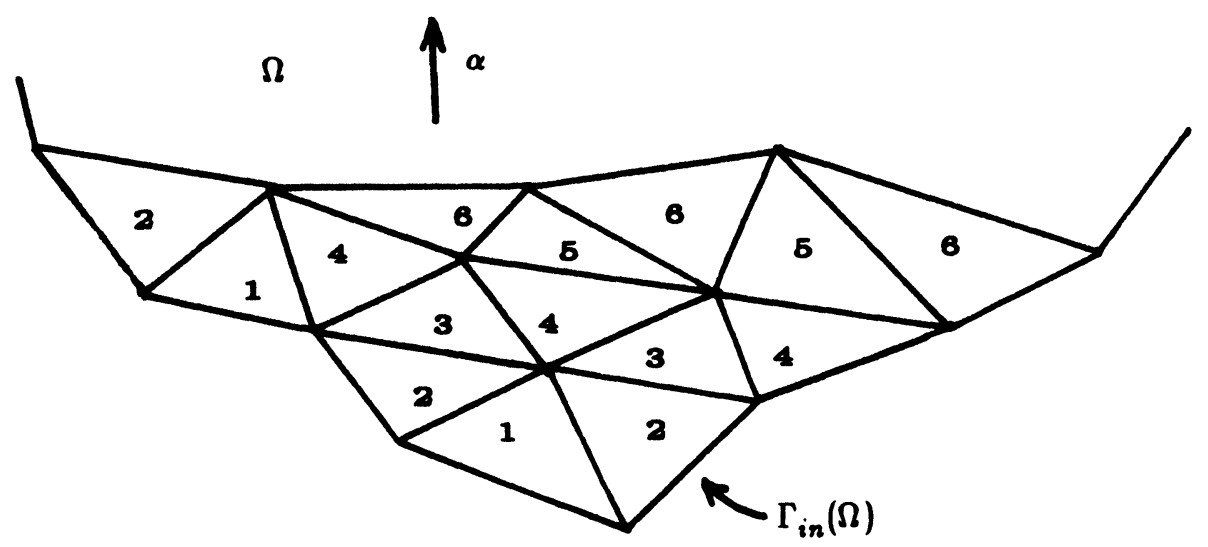

FIG. 2.2 
H3: The ratio of the maximum to minimum triangle sides within $\Delta_{h}$ is uniformly bounded.

H4: The triangles in $\Delta_{h}$ can be partitioned into $O\left(h^{-1}\right)$ layers (where layers are as defined above).

For the sake of brevity and to avoid the introduction of additional variables, we shall use the notation $u_{\alpha}$ to mean $\nabla u \cdot \alpha$ when $\alpha$ is any unit vector. Equivalently, one may think of $u_{\alpha}$ as a partial derivative of $u$ with respect to a coordinate in the direction $\alpha$, provided the second variable on which $u$ depends is constant in the direction $\alpha$. In this notation, we state an integration by parts formula

$$
\int_{T} v_{\alpha} w=\int_{\Gamma(T)} v w \alpha \cdot n-\int_{T} v w_{\alpha}-\int_{T}(\nabla \cdot \alpha) v w
$$

which will be used several times in the paper.

Finally, we shall use the symbol $C$ to denote a generic constant, depending at most on the coefficients $\alpha$ and $\beta$ in (1) and the bounds in assumptions H1-H4.

3. Existence and uniqueness. In order to establish the results in this section, it will be convenient to introduce a reference triangle $\hat{T}$ with vertices $\hat{a}_{1}=(1,0), \hat{a}_{2}=(0,1)$, and $\hat{a}_{3}=(0,0)$. The reference triangle $\hat{T}$ can be mapped into the triangle $T$ by the affine transformation

$$
\left(\begin{array}{l}
x \\
y
\end{array}\right)=\mathbf{F}\left(\begin{array}{l}
\hat{x} \\
\hat{y}
\end{array}\right)+a_{3} .
$$

The matrix $\mathbf{F}$ can be written

$$
\mathbf{F}=\left(\left|\Gamma_{2}\right| \tau_{2},-\left|\Gamma_{1}\right| \tau_{1}\right)
$$

where $\left|\Gamma_{i}\right|$ is the length of $\Gamma_{i}$. Defining $\hat{v}_{h}(\hat{x}, \hat{y})=v(x, y)$ (for an arbitrary $v$ defined on $T$ ) and

$$
\hat{\nabla}=\left(\begin{array}{l}
\partial / \partial \hat{x} \\
\partial / \partial \hat{y}
\end{array}\right)
$$

we have

$$
\alpha \cdot \nabla u_{h}=A \cdot \hat{\nabla} \hat{u}_{h}
$$

where

$$
A=\mathbf{F}^{-1} \alpha \quad \text { and } \quad \mathbf{F}^{-1}=\frac{1}{n_{1} \cdot \tau_{2}}\left(\begin{array}{c}
n_{1}^{t} /\left|\Gamma_{2}\right| \\
n_{2}^{t} /\left|\Gamma_{1}\right|
\end{array}\right) .
$$

Transforming (3) and (4) to $\hat{T}$, we obtain

$$
\int_{\hat{T}}\left(A \cdot \hat{\nabla} \hat{u}_{h}+\hat{\beta} \hat{u}_{h}-\hat{f}\right) \hat{v}_{h} d \hat{x} d \hat{y}=0 \quad \text { for all } \hat{v}_{h} \in \mathbf{P}_{n-\rho}(\hat{T})
$$

where $\rho$ is the number of inflow sides that $\hat{T}$ has. In $\hat{T}$, we express $\hat{u}_{h}$ in the form

$$
\hat{u}_{h}(\hat{x}, \hat{y})=\sum_{j=1}^{\sigma_{n}} \hat{u}_{h}\left(\hat{P}_{j}\right) \hat{\phi}_{j}(\hat{x}, \hat{y})
$$

where $\hat{P}_{j}$ are the usual equispaced nodes for $n$th degree interpolation in $\hat{T}$ and $\hat{\phi}_{j}(\hat{x}, \hat{y})$ are the corresponding Lagrange basis functions for $\mathbf{P}_{n}(\hat{T})$. We then require that $(6)$ 
be satisfied for each of the analogous basis functions $\hat{\psi}_{i}(\hat{x}, \hat{y})$ for $\mathbf{P}_{n-\rho}(\hat{T})$. The resulting linear algebraic system is equivalent to the following:

$$
\begin{aligned}
& \sum_{j=1}^{\sigma_{n}} h\left(A \cdot \hat{\nabla} \hat{\phi}_{j}+\hat{\beta} \hat{\phi}_{j}, \hat{\psi}_{i}\right) \hat{T} \hat{u}_{h}\left(\hat{P}_{j}\right)=h\left(\hat{f}, \hat{\psi}_{i}\right)_{\hat{T}}, \quad i=1, \cdots, \sigma_{n-\rho}, \\
& \hat{u}_{h}\left(\hat{P}_{j}\right)=\text { given value for } \hat{P}_{j} \in \Gamma_{i n}(\hat{T})
\end{aligned}
$$

or, in matrix form,

$$
\mathbf{K} \hat{u}_{h}=b .
$$

The above are $\sigma_{n}$ equations in as many variables, the inflow yielding a total of $n+1$ or $2 n+1$ conditions, depending on whether $T$ is of type I or II.

Now let $Q$ denote the centroid of $T, \alpha_{0}=\alpha(Q)$, and $A_{0}=F^{-1} \alpha_{0}$. The coefficient matrix $\mathbf{K}$ can then be written

$$
\mathbf{K}=\mathbf{K}_{0}+h \mathbf{K}_{1}
$$

where $\mathbf{K}_{0}$ corresponds to $\alpha=\alpha_{0}$ and $\beta=0$. Note that $h A_{0}$ involves ratios of sides of triangles in $\Delta_{h}$ which, by $\mathrm{H} 3$, are uniformly bounded, and that $\left|\alpha(x)-\alpha_{0}\right| \leqq C h\|\alpha\|_{1, \infty}$ for $x \in T$. Thus both $\mathbf{K}_{0}$ and $\mathbf{K}_{1}$ are uniformly bounded over all triangles. We next show that $\mathbf{K}_{0}$ is invertible under assumption $\mathrm{H} 2$. It will then follow that $\mathbf{K}_{0}^{-1}$ is uniformly bounded and that Problem $\mathbf{P}_{\mathbf{h}}$ has a unique solution for sufficiently small $h$.

Establishing the invertibility of $\mathbf{K}_{0}$ is equivalent to showing that the only solution to (3) or (4) with $\beta=f=0, \alpha$ replaced by $\alpha_{0}$, and $u_{h}=0$ on $\Gamma_{i n}(T)$ is $u_{h} \equiv 0$. We now do this.

For type I triangles, we take $v_{h}=\left(u_{h}\right)_{\alpha_{0}} \in \mathbf{P}_{n-1}(T)$, and infer that $\left(u_{h}\right)_{\alpha_{0}}=0$. This, together with $u_{h}=0$ on $\Gamma_{i n}(T)$, implies that $u_{h} \equiv 0$ in $T$. (We have assumed that $h$ is sufficiently small that replacement of $\alpha$ by $\alpha_{0}$ does not alter the inflow and outflow sides of $T$.)

For type II triangles, we note that $u_{h}=0$ on $\Gamma_{i n}(T)$ implies that $u_{h}$ can be written in the form $u_{h}=\xi \eta w_{h}$ where $\xi$ and $\eta$ are coordinates along the two inflow sides, with $\xi=\eta=0$ at $a_{3}, \xi, \eta \geqq 0$ in $T$, and $w_{h} \in \mathbf{P}_{n-2}(T)$. Taking $v_{h}=w_{h}$ in (4), we use the integration by parts formula (5) to obtain

$$
\begin{aligned}
0 & =\left(\left(u_{h}\right)_{\alpha_{0}}, w_{h}\right)_{T}=\left(\xi \eta\left(w_{h}\right)_{\alpha_{0}}, w_{h}\right)_{T}+\left((\xi \eta)_{\alpha_{0}} w_{h}, w_{h}\right)_{T} \\
& =\frac{1}{2}\left(\xi \eta,\left(w_{h}^{2}\right)_{\alpha_{0}}\right)_{T}+\left((\xi \eta)_{\alpha_{0}}, w_{h}^{2}\right)_{T} \\
& =\frac{1}{2} \int_{\Gamma_{\text {out }}(T)} w_{h}^{2} \xi \eta \alpha_{0} \cdot n d \Gamma+\frac{1}{2}\left((\xi \eta)_{\alpha_{0}}, w_{h}^{2}\right)_{T} .
\end{aligned}
$$

Now $(\xi \eta)_{\alpha_{0}}$ is positive in $T$ and $\xi \eta \alpha_{0} \cdot n$ is nonnegative on $\Gamma_{o u t}(T)$. Hence $w_{h} \equiv 0$ in $T$ and $u_{h} \equiv 0$ in $T$. We have thus established the following result.

Lemma 3.1. There exists a constant $h_{0}$ such that for all $h \leqq h_{0}$, Problem $\mathbf{P}_{\mathrm{h}}$ has a unique solution.

We shall henceforth implicitly assume that $h \leqq h_{0}$, so that the approximate solution is well defined. We now establish several estimates applying over a single triangle $T$. They will be used later in the derivation of the main stability result.

LEMMA 3.2. Let $u_{h} \in \mathbf{P}_{n}(T)$ satisfy (3) or (4). Then

$$
\left\|u_{h}\right\|_{T} \leqq C\left\{h^{1 / 2}\left|u_{h}\right|_{\Gamma_{i n}(T)}+h\|f\|_{T}\right\}
$$


and

$$
\left\|\nabla u_{h}\right\|_{T} \leqq C\left\{h^{1 / 2}\left|\frac{d u_{h}}{d \tau}\right|_{\Gamma_{i n}(T)}+h^{1 / 2}\|\beta\|_{\infty, T}\left|u_{h}\right|_{\Gamma_{i n}(T)}+\|f\|_{T}\right\}
$$

where $\tau$ denotes arclength along $\Gamma_{\text {in }}(T)$.

Proof. From Lemma 3.1 and (7) and (8), it immediately follows that

$$
\left\|\hat{u}_{h}\right\|_{\hat{T}} \leqq C\left\{\left|\hat{u}_{h}\right|_{\Gamma_{\text {in }}(\hat{T})}+h\|\hat{f}\|_{\hat{T}}\right\} \text {. }
$$

Inequality (9) follows from (11) by transforming coordinates from $\hat{T}$ to $T$. To prove (10), let $P_{0}$ be a point on the inflow to $T$ and write $u_{h}=u_{h}\left(P_{0}\right)+v_{h}$. Then

$$
\left|v_{h}\right|_{\Gamma_{i n}(T)} \leqq C h\left|\frac{d v_{h}}{d \tau}\right|_{\Gamma_{i n}(T)}=C h\left|\frac{d u_{h}}{d \tau}\right|_{\Gamma_{i n}(T)}
$$

and

$$
\left\|\nabla u_{h}\right\|_{T}=\left\|\nabla v_{h}\right\|_{T} \leqq C h^{-1}\left\|v_{h}\right\|_{T} .
$$

Now $v_{h} \in \mathbf{P}_{n}(T)$ satisfies (3) or (4) with $f$ replaced by $f-\beta u_{h}\left(P_{0}\right)$. Applying the first part of the lemma, we obtain

$$
\begin{aligned}
\left\|v_{h}\right\|_{T} & \leqq C\left\{h^{1 / 2}\left|v_{h}\right|_{\Gamma_{i n}(T)}+h\left\|f-\beta u_{h}\left(P_{0}\right)\right\|_{T}\right\} \\
& \leqq C\left\{h^{3 / 2}\left|\frac{d u_{h}}{d \tau}\right|_{\Gamma_{i n}(T)}+h\|f\|_{T}+h\|\beta\|_{\infty, T}\left\|u_{h}\left(P_{0}\right)\right\|_{T}\right\} \\
& \leqq C\left\{h^{3 / 2}\left|\frac{d u_{h}}{d \tau}\right|_{\Gamma_{i n}(T)}+h\|f\|_{T}+h^{3 / 2}\|\beta\|_{\infty, T}\left|u_{h}\right|_{\Gamma_{i n}(T)}\right\} .
\end{aligned}
$$

The result follows immediately.

Lemma 3.3. Let $T$ be a type II triangle and $\phi_{h} \in \mathbf{P}_{n}(T)$. Then

$$
\left\|\left(\phi_{h}\right)_{\alpha}\right\|_{T} \leqq C\left\{h^{1 / 2}\left|\left(\phi_{h}\right)_{\alpha}\right|_{\Gamma_{\text {out }}(T)}+\left\|P_{n-2}\left(\phi_{h}\right)_{\alpha}\right\|_{T}+h\left\|\nabla \phi_{h}\right\|_{T}\right\} \text {. }
$$

Proof. We first prove the result for the case $\alpha=\alpha_{0}$, a constant vector. Here $v \equiv\left(\phi_{h}\right)_{\alpha_{0}} \in \mathbf{P}_{n-1}(T)$. Moreover, if $v=0$ on $\Gamma_{\text {out }}(T)$ and $P_{n-2} v=0$, then $v \equiv 0$ in $T$. This can be shown by writing $v=p(\tau)+s q(s, \tau)$, where $\tau$ is a coordinate measured along $\Gamma_{\text {out }}(T)$ and $s$ is a coordinate in the direction of $\alpha_{0}$ with $s=0$ on $\Gamma_{\text {out }}(T)$. Then $v=0$ on $\Gamma_{\text {out }}(T)$ implies that $p(\tau)=0$ and $P_{n-2} v=0$ therefore implies that

$$
\int_{T} s q w d x d y=0 \text { for all } w \in \mathbf{P}_{n-2}(T) .
$$

By taking $w=q \in \mathbf{P}_{n-2}(T)$, we conclude that $q \equiv 0$ in $T$. Thus $v \equiv 0$ in $T$. In the reference triangle $\hat{T}$, we therefore have

$$
\|\hat{v}\|_{\hat{T}} \leqq C\left\{|\hat{v}|_{\Gamma_{\text {out }}(\hat{T})}+\left\|P_{n-2} \hat{v}\right\|_{\hat{T}}\right\},
$$

which, when transformed back to the original triangle, becomes

$$
\left\|\left(\phi_{h}\right)_{\alpha_{0}}\right\|_{T} \leqq C\left\{h^{1 / 2} \mid\left(\phi_{h}\right)_{\alpha_{0} \Gamma_{\text {our }}(T)}+\left\|P_{n-2}\left(\phi_{h}\right)_{\alpha_{0}}\right\|_{T}\right\} .
$$

Now suppose $\alpha$ is not constant. Using (13), we obtain

$$
\begin{aligned}
\left\|\left(\phi_{h}\right)_{\alpha}\right\|_{T} \leqq\left\|\left(\phi_{h}\right)_{\alpha_{0}}\right\|_{T}+\left\|\left(\alpha-\alpha_{0}\right) \cdot \nabla \phi_{h}\right\|_{T} \\
\leqq C\left\{h^{1 / 2}\left|\left(\phi_{h}\right)_{\alpha}\right|_{\Gamma_{o u t}(T)}+\left\|P_{n-2}\left(\phi_{h}\right)_{\alpha}\right\|_{T}\right. \\
\left.\quad+h^{1 / 2}\left|\left(\alpha_{0}-\alpha\right) \cdot \nabla \phi_{h}\right|_{\Gamma_{\text {our }}(T)}+\left\|P_{n-2}\left(\left[\alpha_{0}-\alpha\right] \cdot \nabla \phi_{h}\right)\right\|_{T}\right\} \\
\quad+\left\|\left(\alpha-\alpha_{0}\right) \cdot \nabla \phi_{h}\right\|_{T} .
\end{aligned}
$$

Inequality (12) now follows by standard estimates. 
4. Basic identities. The test function $v_{h}=-\left(u_{h}\right)_{\tau_{1} \tau_{2}}$ in (3) and (4) will play a key role in our stability analysis. (Recall that $\tau_{1}$ and $\tau_{2}$ are the tangents to the two outflow sides of a type I triangle or the two inflow sides of a type II triangle.) In this section, we derive expressions for the two terms in the integral

$$
\left(u_{\alpha}+\beta u,-u_{\tau_{1} \tau_{2}}\right)_{T} .
$$

To facilitate the exposition, we state some obvious identities relating $\alpha=\left(\alpha_{1}, \alpha_{2}\right)^{t}$ and $\tau_{i}$ and $n_{i}$, the unit tangential and normal vectors to the sides $\Gamma_{i}$ of a triangle $T$ (as depicted in Fig. 2.1). Let $\gamma=\left(-\alpha_{2}, \alpha_{1}\right)^{t}$. Then

$$
\begin{array}{lll}
\text { (a) } \tau_{i} \cdot n_{j}=-\tau_{j} \cdot n_{i}, & i, j=1,2,3, \\
\text { (b) } \tau_{i} \cdot \gamma=n_{i} \cdot \alpha, & i=1,2,3, \\
\text { (c) } \gamma \cdot n_{i}=-\alpha \cdot \tau_{i}, & i=1,2,3 .
\end{array}
$$

In addition, the following identity is also valid for any unit vector $\alpha=\left(\alpha_{1}, \alpha_{2}\right)^{t}$.

LEMMA 4.1.

$$
\left(\alpha \cdot n_{2}\right)\left(\tau_{1} \cdot n_{3}\right)+\left(\alpha \cdot n_{1}\right)\left(\tau_{3} \cdot n_{2}\right)+\left(\alpha \cdot n_{3}\right)\left(\tau_{2} \cdot n_{1}\right)=0 .
$$

Proof. Let $\xi_{i}$ denote the angle between $\alpha$ and $n_{i}$, measured counterclockwise from $\alpha$. Since $\tau_{i}$ may be obtained by rotating $n_{i}$ through an angle $\pi / 2$,

$$
\tau_{i} \cdot n_{j}=\cos \left[\left(\xi_{i}+\pi / 2\right)-\xi_{j}\right]=\sin \left(\xi_{j}-\xi_{i}\right) .
$$

Thus the lemma may be recast as

$$
\cos \xi_{2} \sin \left(\xi_{3}-\xi_{1}\right)+\cos \xi_{1} \sin \left(\xi_{2}-\xi_{3}\right)+\cos \xi_{3} \sin \left(\xi_{1}-\xi_{2}\right)=0
$$

which can be verified by expanding the sines in the above formula.

We shall now derive the desired identities.

LEMMA 4.2. For any unit vector $\alpha$ and any twice differentiable function $u$ :

$$
\begin{aligned}
\int_{T} u_{\alpha}\left(-u_{\tau_{1} \tau_{2}}\right) d x d y= & \frac{1}{2} \int_{\Gamma(T)} \frac{\left(\alpha \cdot n_{1}\right)\left(\alpha \cdot n_{2}\right)}{\alpha \cdot n} u_{\tau}^{2} d \tau-\frac{1}{2} \int_{\Gamma_{3}} \frac{\left(\tau_{1} \cdot n_{3}\right)\left(\tau_{2} \cdot n_{3}\right)}{\alpha \cdot n_{3}} u_{\alpha}^{2} d \tau \\
& +\frac{1}{2} \int_{T}\left\{\left(A_{1}\right)_{\tau_{2}} u_{\tau_{1}}^{2}+\left(A_{2}\right)_{\tau_{1}} u_{\tau_{2}}^{2}\right\} d x d y
\end{aligned}
$$

where $\left(A_{1}, A_{2}\right)=\left(1 / \tau_{1} \cdot n_{2}\right)\left(\alpha \cdot n_{2},-\alpha \cdot n_{1}\right)$.

Proof. We first write $u_{\alpha}$ in terms of derivatives in the directions of $\tau_{1}$ and $\tau_{2}$. From

$$
\left(\begin{array}{l}
u_{x} \\
u_{y}
\end{array}\right)=\left(\begin{array}{l}
\tau_{1}^{t} \\
\tau_{2}^{t}
\end{array}\right)^{-1}\left(\begin{array}{l}
u_{\tau_{1}} \\
u_{\tau_{2}}
\end{array}\right)=\frac{1}{\tau_{2} \cdot n_{1}}\left(-n_{2}, n_{1}\right)\left(\begin{array}{l}
u_{\tau_{1}} \\
u_{\tau_{2}}
\end{array}\right) \text {, }
$$

it follows that

$$
u_{\alpha}=A_{1} u_{\tau_{1}}+A_{2} u_{\tau_{2}},
$$

with $A_{1}$ and $A_{2}$ as defined in the statement of the lemma. Hence, using the integration by parts formula (5) and (15a) from the previous page, we obtain

$$
\begin{aligned}
\int_{T} u_{\alpha}\left(-u_{\tau_{1} \tau_{2}}\right) d x d y= & -\frac{1}{2} \int_{T}\left\{A_{1}\left(u_{\tau_{1}}^{2}\right)_{\tau_{2}}+A_{2}\left(u_{\tau_{2}}^{2}\right)_{\tau_{1}}\right\} d x d y \\
= & \frac{1}{2} \int_{\Gamma_{1}}\left(\alpha \cdot n_{2}\right) u_{\tau_{1}}^{2} d \tau+\frac{1}{2} \int_{\Gamma_{2}}\left(\alpha \cdot n_{1}\right) u_{\tau_{2}}^{2} d \tau \\
& -\frac{1}{2} \int_{\Gamma_{3}}\left\{A_{1} u_{\tau_{1}}^{2}\left(\tau_{2} \cdot n_{3}\right)+A_{2} u_{\tau_{2}}^{2}\left(\tau_{1} \cdot n_{3}\right)\right\} d \tau
\end{aligned}
$$




$$
+\frac{1}{2} \int_{T}\left\{\left(A_{1}\right)_{\tau_{2}} u_{\tau_{1}}^{2}+\left(A_{2}\right)_{\tau_{1}} u_{\tau_{2}}^{2}\right\} d x d y
$$

We next write $u_{\tau_{1}}$ and $u_{\tau_{2}}$ in terms of the directional derivatives $u_{\tau_{3}}$ and $u_{\alpha}$. Let $\gamma$ be the unit vector $\left(-\alpha_{2}, \alpha_{1}\right)^{t}$. Then

$$
\left(\begin{array}{l}
u_{x} \\
u_{y}
\end{array}\right)=\left(\begin{array}{c}
\tau_{3}^{t} \\
\alpha^{t}
\end{array}\right)^{-1}\left(\begin{array}{l}
u_{\tau_{3}} \\
u_{\alpha}
\end{array}\right)=\frac{1}{\tau_{3} \cdot \gamma}\left(\gamma, n_{3}\right)\left(\begin{array}{l}
u_{\tau_{3}} \\
u_{\alpha}
\end{array}\right)
$$

and

$$
\left(\begin{array}{l}
u_{\tau_{1}} \\
u_{\tau_{2}}
\end{array}\right)=\left(\begin{array}{c}
\tau_{1}^{t} \\
\tau_{2}^{t}
\end{array}\right)\left(\begin{array}{l}
u_{x} \\
u_{y}
\end{array}\right)=\frac{1}{\tau_{3} \cdot \gamma}\left(\begin{array}{cc}
\tau_{1} \cdot \gamma & \tau_{1} \cdot n_{3} \\
\tau_{2} \cdot \gamma & \tau_{2} \cdot n_{3}
\end{array}\right)\left(\begin{array}{c}
u_{\tau_{3}} \\
u_{\alpha}
\end{array}\right) .
$$

Using this result and the definitions of $A_{1}$ and $A_{2}$, we now rewrite the integrand along $\Gamma_{3}$ as follows:

$$
\begin{aligned}
& A_{1} u_{\tau_{1}}^{2}\left(\tau_{2} \cdot n_{3}\right)+A_{2} u_{\tau_{2}}^{2}\left(\tau_{1} \cdot n_{3}\right) \\
& \begin{array}{l}
=\frac{1}{\left(\tau_{3} \cdot \gamma\right)^{2}\left(\tau_{1} \cdot n_{2}\right)} \\
\quad \cdot\left\{\left[\left(\alpha \cdot n_{2}\right)\left(\tau_{2} \cdot n_{3}\right)\left(\tau_{1} \cdot \gamma\right)^{2}-\left(\alpha \cdot n_{1}\right)\left(\tau_{1} \cdot n_{3}\right)\left(\tau_{2} \cdot \gamma\right)^{2}\right] u_{\tau_{3}}^{2}\right. \\
\quad+\left[\left(\alpha \cdot n_{2}\right)\left(\tau_{2} \cdot n_{3}\right)\left(\tau_{1} \cdot n_{3}\right)^{2}-\left(\alpha \cdot n_{1}\right)\left(\tau_{1} \cdot n_{3}\right)\left(\tau_{2} \cdot n_{3}\right)^{2}\right] u_{\alpha}^{2} \\
\quad+2\left[\left(\alpha \cdot n_{2}\right)\left(\tau_{2} \cdot n_{3}\right)\left(\tau_{1} \cdot \gamma\right)\left(\tau_{1} \cdot n_{3}\right)\right. \\
\left.\left.\quad-\left(\alpha \cdot n_{1}\right)\left(\tau_{1} \cdot n_{3}\right)\left(\tau_{2} \cdot \gamma\right)\left(\tau_{2} \cdot n_{3}\right)\right] u_{\tau_{3}} u_{\alpha}\right\} .
\end{array}
\end{aligned}
$$

Using Lemma 4.1 and (15b), we then obtain

$$
A_{1} u_{\tau_{1}}^{2}\left(\tau_{2} \cdot n_{3}\right)+A_{2} u_{\tau_{2}}^{2}\left(\tau_{1} \cdot n_{3}\right)=\frac{1}{\alpha \cdot n_{3}}\left\{-\left(\alpha \cdot n_{1}\right)\left(\alpha \cdot n_{2}\right) u_{\tau_{3}}^{2}+\left(\tau_{1} \cdot n_{3}\right)\left(\tau_{2} \cdot n_{3}\right) u_{\alpha}^{2}\right\}
$$

Lemma 4.2 now follows by combining terms.

Lemma 4.3. Let $T \in\left\{\Delta_{h}\right\}$, and for $P \in \Gamma$ let $\theta(P)$ be the angle from $\alpha(P)$ to the local tangent vector $\tau$, measured counterclockwise. Then

$$
\begin{aligned}
\int_{T} \beta u\left(-u_{\tau_{1} \tau_{2}}\right) d x d y= & \int_{T} \beta u_{\tau_{1}} u_{\tau_{2}} d x d y-\int_{\Gamma(T)} \beta\left(\alpha \cdot n_{1}\right)\left(\alpha \cdot n_{2}\right) \cot \theta u u_{\tau} d \tau \\
& -\int_{\Gamma_{3}(T)} \beta \frac{\left(\tau_{2} \cdot n_{3}\right)\left(\tau_{1} \cdot n_{3}\right)}{\alpha \cdot n_{3}} u u_{\alpha} d \tau-\frac{1}{2} \int_{\Gamma(T)} \rho_{\tau} u^{2} d \tau \\
& +\int_{T} u\left[\left(\beta \phi_{1}\right)_{\tau_{2}} u_{\tau_{1}}+\left(\beta \phi_{2}\right)_{\tau_{1}} u_{\tau_{2}}\right] d x d y
\end{aligned}
$$

where

$$
\phi_{1}=\frac{\left(\alpha \cdot n_{2}\right)\left(r_{1} \cdot n_{3}\right)}{\left(\alpha \cdot n_{3}\right)\left(\tau_{1} \cdot n_{2}\right)}, \quad \phi_{2}=\frac{\left(\alpha \cdot n_{1}\right)\left(\tau_{2} \cdot n_{3}\right)}{\left(\alpha \cdot n_{3}\right)\left(\tau_{2} \cdot n_{1}\right)}
$$

and

$$
\rho=\frac{\beta\left(\alpha \cdot n_{1}\right)\left(\alpha \cdot n_{2}\right)\left(\alpha \cdot \tau_{3}\right)}{\alpha \cdot n_{3}}
$$


Proof. It follows immediately from Lemma 4.1 and the definitions of $\phi_{1}$ and $\phi_{2}$ that $\phi_{1}+\phi_{2}=1$. Applying the integration by parts formula (5), we obtain

$$
\begin{aligned}
\int_{T} \beta u\left(-u_{\tau_{1} \tau_{2}}\right) d x d y= & \int_{T} \phi_{1} \beta u\left(-u_{\tau_{1} \tau_{2}}\right) d x d y+\int_{T} \phi_{2} \beta u\left(-u_{\tau_{2} \tau_{1}}\right) d x d y \\
= & -\int_{\Gamma} \beta u\left[\phi_{1} u_{\tau_{1}}\left(\tau_{2} \cdot n\right)+\phi_{2} u_{\tau_{2}}\left(\tau_{1} \cdot n\right)\right] d \tau \\
& +\int_{T} \beta u_{\tau_{2}} u_{\tau_{1}} d x d y+\int_{T} u\left[\left(\beta \phi_{1}\right)_{\tau_{2}} u_{\tau_{1}}+\left(\beta \phi_{2}\right)_{\tau_{1}} u_{\tau_{2}}\right] d x d y .
\end{aligned}
$$

We now derive another form for the integral around $\Gamma(T)$. Using the definitions of $\phi_{1}$ and $\phi_{2},(15 b)$, and formula (16), we have

$$
\phi_{1} u_{\tau_{1}}\left(\tau_{2} \cdot n_{3}\right)+\phi_{2} u_{\tau_{2}}\left(\tau_{1} \cdot n_{3}\right)=\frac{\left(\tau_{1} \cdot n_{3}\right)\left(\tau_{2} \cdot n_{3}\right)}{\alpha \cdot n_{3}} u_{\alpha}
$$

We also have the following identity for $i=1,2$ :

$$
\frac{\tau_{i} \cdot n_{3}}{\left(\alpha \cdot n_{3}\right)\left(\alpha \cdot n_{i}\right)}=\frac{\tau_{i} \cdot \alpha}{\alpha \cdot n_{i}}-\frac{\alpha \cdot \tau_{3}}{\alpha \cdot n_{3}}
$$

This may be shown by writing $\tau_{i}=\left(\tau_{i} \cdot \alpha\right) \alpha+\left(\tau_{i} \cdot \gamma\right) \gamma$, and then applying (15b) and (15c).

Combining the preceding formulas, we obtain

$$
\begin{aligned}
-\int_{\Gamma} \beta u\left[\phi_{1} u_{\tau_{1}}\left(\tau_{2} \cdot n\right)+\phi_{2} u_{\tau_{2}}\left(\tau_{1} \cdot n\right)\right] d \tau \\
=-\int_{\Gamma_{3}} \beta \frac{\left(\tau_{1} \cdot n_{3}\right)\left(\tau_{2} \cdot n_{3}\right)}{\alpha \cdot n_{3}} u u_{\alpha} d \tau \\
-\int_{\Gamma_{1} \cup \Gamma_{2}} \beta\left(\alpha \cdot n_{1}\right)\left(\alpha \cdot n_{2}\right) \frac{\alpha \cdot \tau}{\alpha \cdot n} u u_{\tau} d \tau+\int_{\Gamma_{1} \cup \Gamma_{2}} \rho u u_{\tau} d \tau
\end{aligned}
$$

where $\rho$ is as defined in the statement of the lemma. An integration by parts yields for the last term

$$
\begin{aligned}
\int_{\Gamma_{1} \cup \Gamma_{2}} \rho u u_{\tau} d \tau & =\frac{1}{2}\left\{\left[\rho u^{2}\right]_{a_{2}}^{a_{3}}+\left[\rho u^{2}\right]_{a_{3}}^{a_{1}}-\int_{\Gamma_{1} \cup \Gamma_{2}} \rho_{\tau} u^{2} d \tau\right\} \\
& =-\int_{\Gamma_{3}} \rho u u_{\tau} d \tau-\frac{1}{2} \int_{\Gamma} \rho_{\tau} u^{2} d \tau .
\end{aligned}
$$

The lemma now follows by combining results and observing that along $\Gamma_{i}$

$$
\frac{\alpha \cdot \tau_{i}}{\alpha \cdot n_{i}}=\frac{\cos \theta_{i}}{\cos \left(\theta_{i}-\pi / 2\right)}=\cot \theta_{i}
$$

where $\theta_{i}(P)$ denotes the angle from $\alpha(P)$ to $\tau_{i}$, measured counterclockwise.

5. Stability. In this section we derive the basic stability estimates for Problem $\mathbf{P}_{\mathbf{h}}$. These will be used to obtain error estimates in the next section. The norm in which we obtain stability is a weighted sum of $L^{2}$ norms of $u_{h}$ and its tangential derivative 
taken along fronts $F_{j}$, which describe the forward boundary of the solution after it has progressed through the first $j$ layers. More specifically, we define

$$
\begin{aligned}
& F_{0}=\Gamma_{\text {in }}(\Omega), \\
& F_{j}=F_{j-1} \cup \Gamma_{\text {out }}\left(S_{j}\right)-\Gamma_{\text {in }}\left(S_{j}\right), \quad j=1,2, \cdots .
\end{aligned}
$$

The main result of this section will be the following stability theorem.

THEOREM 5.1. If $u_{h}$ is the solution of Problem $\mathbf{P}_{\mathrm{h}}$, then for $h$ sufficiently small

$$
\begin{aligned}
& \int_{F_{j}}\left\{\frac{h^{3 / 2}\left(d u_{h} / d \tau\right)^{2}}{|\alpha \cdot n|}+u_{h}^{2}|\alpha \cdot n|\right\} d \tau+M h^{1 / 2}\left\|\left(u_{h}\right)_{\alpha}\right\|_{\Omega_{j}}^{2} \\
& \leqq C\left\{h^{1 / 2}\|f\|_{\Omega_{j}}^{2}+h^{-1 / 2}\left\|P_{n-2} f\right\|_{\Omega_{j}}^{2}+\int_{F_{0}}\left\{\frac{h^{3 / 2}\left(d u_{h} / d \tau\right)^{2}}{|\alpha \cdot n|}+u_{h}^{2}|\alpha \cdot n|\right\} d \tau\right\}
\end{aligned}
$$

where $\Omega_{j}=\cup_{k \leqq j} S_{k}$ and $M$ is a positive constant.

To prove this theorem, we first develop local stability results applicable over a single triangle. This is complicated somewhat by the fact that the two different types of triangles require different treatment. For each, we shall obtain a bound on the growth of $d u_{h} / d \tau$ from the identities in the previous section. When these are combined suitably with bounds on the growth of $u_{h}$, the desired stability result is obtained. We note the factor $h^{3 / 2}$, which appears in Theorem 5.1, rather than $h^{2}$ which might be expected from approximation theoretic considerations.

The next two lemmas bound the growth in $u_{h}$ over the two types of triangles.

LEMMA 5.1. If $T$ is a type I triangle and $u_{h}$ satisfies (3) in $T$, then

$$
\int_{\Gamma(T)} u_{h}^{2} \alpha \cdot n d \tau \leqq C\left\{h^{1 / 2}\|f\|_{T}^{2}+\left\|P_{n-2} f\right\|_{T}^{2}+h^{3 / 2}\left\|\nabla u_{h}\right\|_{T}^{2}+\left\|u_{h}\right\|_{T}^{2}\right\} .
$$

Proof. Omitting the subscript $T$ on the norms and inner products which follow, we apply $(5)$ to $\left(\left(u_{h}\right)_{\alpha}, u_{h}\right)$. This yields

$$
\begin{aligned}
\frac{1}{2} \int_{\Gamma} u_{h}^{2} \alpha \cdot n d \tau= & \frac{1}{2}\left(u_{h}^{2}, \nabla \cdot \alpha\right)+\left(\left(u_{h}\right)_{\alpha}, u_{h}\right) \\
= & \frac{1}{2}\left(u_{h}^{2}, \nabla \cdot \alpha\right)+\left(\left(u_{h}\right)_{\alpha},\left(I-P_{n-1}\right) u_{h}\right)+\left(\left(u_{h}\right)_{\alpha}, P_{n-1} u_{h}\right) \\
= & \frac{1}{2}\left(u_{h}^{2}, \nabla \cdot \alpha\right)+\left(\left(\alpha-\alpha_{0}\right) \cdot \nabla u_{h},\left(I-P_{n-1}\right) u_{h}\right)-\left(f,\left(I-P_{n-1}\right) u_{h}\right) \\
& \quad+\left(f,\left(I-P_{n-2}\right) u_{h}\right)+\left(f, P_{n-2} u_{h}\right)-\left(\beta u_{h}, P_{n-1} u_{h}\right) .
\end{aligned}
$$

In the last of these equalities, (3) and the fact that $\left(u_{h}\right)_{\alpha_{0}} \in \mathbf{P}_{n-1}(T)$ were used. Applying standard estimates we obtain

$$
\begin{aligned}
\int_{\Gamma} u_{h}^{2} \alpha \cdot n d \tau \leqq C\left\{\left[\|\alpha\|_{1, \infty}+\|\beta\|_{\infty}\right]\left\|u_{h}\right\|^{2}+h^{2}\|\alpha\|_{1, \infty}\left\|\nabla u_{h}\right\|^{2}\right. \\
\left.+h\|f\|\left\|\nabla u_{h}\right\|+\left\|P_{n-2} f\right\|\left\|u_{h}\right\|\right\} \\
\leqq C\left\{h^{1 / 2}\|f\|^{2}+\left\|P_{n-2} f\right\|^{2}+h^{3 / 2}\left\|\nabla u_{h}\right\|^{2}+\left\|u_{h}\right\|^{2}\right\} .
\end{aligned}
$$

LEMMA 5.2. If $T$ is a type II triangle and $u_{h}$ satisfies (4) on $T$, then for any $\varepsilon>0$

$$
\int_{\Gamma(T)} u_{h}^{2} \alpha \cdot n d \tau \leqq \varepsilon h^{3 / 2}\left|\left(u_{h}\right)_{\alpha}\right|_{\Gamma_{o u t}(T)}^{2}+C\left\{\left\|P_{n-2} f\right\|_{T}^{2}+h^{3 / 2}\left\|\nabla u_{h}\right\|_{T}^{2}+\left\|u_{h}\right\|_{T}^{2}\right\}
$$

where $C$ depends on $\varepsilon$. 
Proof. Again omitting the subscript $T$ and using (5), we obtain

$$
\begin{aligned}
\frac{1}{2} \int_{\Gamma} u_{h}^{2} \alpha \cdot n d \tau & =\frac{1}{2}\left(u_{h}^{2}, \nabla \cdot \alpha\right)+\left(\left(u_{h}\right)_{\alpha}, u_{h}\right) \\
& =\frac{1}{2}\left(u_{h}^{2}, \nabla \cdot \alpha\right)+\left(\left(u_{h}\right)_{\alpha},\left(I-P_{n-2}\right) u_{h}\right)+\left(P_{n-2} f, P_{n-2} u_{h}\right)-\left(\beta u_{h}, P_{n-2} u_{h}\right) .
\end{aligned}
$$

It then follows by standard estimates that

$$
\begin{aligned}
\int_{\Gamma} u_{h}^{2} \alpha \cdot n d \tau & \leqq C\left\{\left[\|\alpha\|_{1, \infty}+\|\beta\|_{\infty}+1\right]\left\|u_{h}\right\|^{2}+\left\|P_{n-2} f\right\|^{2}+h\left\|\left(u_{h}\right)_{\alpha}\right\|\left\|\nabla u_{h}\right\|\right\} \\
& \leqq C\left\{\left\|u_{h}\right\|^{2}+\left\|P_{n-2} f\right\|^{2}+\varepsilon h^{1 / 2}\left\|\left(u_{h}\right)_{\alpha}\right\|^{2}+\varepsilon^{-1} h^{3 / 2}\left\|\nabla u_{h}\right\|^{2}\right\} \quad \text { (for any } \varepsilon>0 \text { ). }
\end{aligned}
$$

Using (12) and (4) we obtain

$$
\begin{aligned}
\left\|\left(u_{h}\right)_{\alpha}\right\|^{2} & \leqq C\left\{h\left|\left(u_{h}\right)_{\alpha}\right|_{\Gamma_{\text {out }}(T)}^{2}+\left\|P_{n-2}\left(u_{h}\right)_{\alpha}\right\|^{2}+h^{2}\left\|\nabla u_{h}\right\|^{2}\right\} \\
& \leqq C\left\{h\left|\left(u_{h}\right)_{\alpha}\right|_{\Gamma_{o u t}(T)}^{2}+\left\|P_{n-2} f\right\|^{2}+\left\|u_{h}\right\|^{2}+h^{2}\left\|\nabla u_{h}\right\|^{2}\right\} .
\end{aligned}
$$

Inserting this result in the previous inequality and replacing $\varepsilon$ by $\varepsilon / C$ establishes the lemma.

We now combine Lemmas 4.2, 4.3, 5.1 and 5.2 into a single local stability result. Theorem 5.1 will then be proved by an appropriate application of this local result.

THEOREM 5.2. Suppose $u_{h}$ satisfies (3) on type I triangles and (4) on type II triangles. The following stability results then hold:

(i) For a type I triangle $T$

$$
\begin{aligned}
\int_{\Gamma(T)} & \left\{\frac{h^{3 / 2}\left(d u_{h} / d \tau\right)^{2}}{\alpha \cdot n}+u_{h}^{2} \alpha \cdot n\right\} d \tau-2 h^{3 / 2} \int_{\Gamma(T)} \beta \cot \theta u_{h}\left(u_{h}\right)_{\tau} d \tau \\
& \leqq C\left\{h^{1 / 2}\|f\|_{T}^{2}+h^{-1 / 2}\left\|P_{n-2} f\right\|_{T}^{2}+h \int_{\Gamma_{i n}(T)}\left[h^{3 / 2}\left(u_{h}\right)_{\tau}^{2}+u_{h}^{2}\right] d \tau\right\} .
\end{aligned}
$$

(ii) For a type II triangle $T$

$$
\begin{gathered}
\int_{\Gamma(T)}\left\{\frac{h^{3 / 2}\left(d u_{h} / d \tau\right)^{2}}{\alpha \cdot n}+u_{h}^{2} \alpha \cdot n\right\} d \tau-2 h^{3 / 2} \int_{\Gamma(T)} \beta \cot \theta u_{h}\left(u_{h}\right)_{\tau} d \tau+\Lambda h^{3 / 2}\left|\left(u_{h}\right)_{\alpha}\right|_{\Gamma_{\text {out }}(T)}^{2} \\
\leq C\left\{h^{3 / 2}\|f\|_{T}^{2}+h^{-1 / 2}\left\|P_{n-2} f\right\|_{T}^{2}+h \int_{\Gamma_{i n}(T)}\left[h^{3 / 2}\left(u_{h}\right)_{\tau}^{2}+u_{h}^{2}\right] d \tau\right\}
\end{gathered}
$$

where $\theta$ is the angle defined in Lemma 4.3 and $\Lambda$ is a positive constant, independent of $h$ and $u_{h}$.

Proof. Let $Q$ be the centroid of triangle $T$ and choose $v_{h}=-\left(u_{h}\right)_{\tau_{1} \tau_{2}}$ in (3) and (4). Using Lemmas 4.2 and 4.3, (3) and (4) become

$$
\begin{aligned}
\frac{1}{2} \int_{\Gamma} \frac{\left(\alpha \cdot n_{1}\right)\left(\alpha \cdot n_{2}\right)}{\alpha \cdot n}\left(u_{h}\right)_{\tau}^{2} d \tau-\int_{\Gamma} \beta\left(\alpha \cdot n_{1}\right)\left(\alpha \cdot n_{2}\right) \cot \theta u_{h}\left(u_{h}\right)_{\tau} d \tau \\
\quad-\frac{1}{2} \int_{\Gamma_{3}} \frac{\left(\tau_{1} \cdot n_{3}\right)\left(\tau_{2} \cdot n_{3}\right)}{\alpha \cdot n_{3}}\left[\left(u_{h}\right)_{\alpha}^{2}+2 \beta u_{h}\left(u_{h}\right)_{\alpha}\right] d \tau \\
=-\left\{\int_{T} f\left(u_{h}\right)_{\tau_{1} \tau_{2}} d x d y+R\right\}
\end{aligned}
$$


where

$$
\begin{aligned}
R= & \int_{T} \beta\left(u_{h}\right)_{\tau_{1}}\left(u_{h}\right)_{\tau_{2}} d x d y+\frac{1}{2} \int_{T}\left[\left(A_{1}\right)_{\tau_{2}}\left(u_{h}\right)_{\tau_{1}}^{2}+\left(A_{2}\right)_{\tau_{1}}\left(u_{h}\right)_{\tau_{2}}^{2}\right] d x d y \\
& +\int_{T} u_{h}\left[\left(\beta \phi_{1}\right)_{\tau_{2}}\left(u_{h}\right)_{\tau_{1}}+\left(\beta \phi_{2}\right)_{\tau_{1}}\left(u_{h}\right)_{\tau_{2}}\right] d x d y-\frac{1}{2} \int_{\Gamma}(\rho)_{\tau} u_{h}^{2} d \tau
\end{aligned}
$$

and $A_{1}, A_{2}, \phi_{1}, \phi_{2}$, and $\rho$ are defined in Lemmas 4.2 and 4.3. First note that by standard estimates

$$
\begin{aligned}
\mid R \| & \leqq C\left\{\left\|\nabla u_{h}\right\|_{T}^{2}+\left\|u_{h}\right\|_{T}^{2}+\left|u_{h}\right|_{\Gamma(T)}^{2}\right\} \\
& \leqq C\left\{\left\|\nabla u_{h}\right\|_{T}^{2}+h^{-1}\left\|u_{h}\right\|_{T}^{2}\right\} .
\end{aligned}
$$

Next, since $\left(u_{h}\right)_{\tau_{1} \tau_{2}} \in \mathbf{P}_{n-2}(T)$,

$$
\begin{aligned}
\left|\int_{T} f\left(u_{h}\right)_{\tau_{1} \tau_{2}} d x d y\right| & \leqq C\left\|P_{n-2} f\right\|_{T}\left\|\left(u_{h}\right)_{\tau_{1} \tau_{2}}\right\|_{T} \\
& \leqq C h^{-1}\left\|P_{n-2} f\right\|_{T}\left\|\nabla u_{h}\right\|_{T} \\
& \leqq C\left\{h^{-2}\left\|P_{n-2} f\right\|_{T}^{2}+\left\|\nabla u_{h}\right\|_{T}^{2}\right\} .
\end{aligned}
$$

Using these bounds in (19), then dividing the resulting inequality by $\frac{1}{2}\left(\alpha(Q) \cdot n_{1}\right)$. $\left(\alpha(Q) \cdot n_{2}\right)$ (a positive quantity by hypothesis $H 2$ ), we obtain

$$
\begin{aligned}
\int_{\Gamma}(1+\sigma) & \frac{\left(u_{h}\right)_{\tau}^{2}}{\alpha \cdot n} d \tau-2 \int_{\Gamma} \beta(1+\sigma) \cot \theta u_{h}\left(u_{h}\right)_{\tau} d \tau \\
& +\int_{\Gamma_{3}} \omega\left\{\left(u_{h}\right)_{\alpha}^{2}+2 \beta u_{h}\left(u_{h}\right)_{\alpha}\right\} d \tau \\
\leqq & C\left\{\left\|\nabla u_{h}\right\|_{T}^{2}+h^{-1}\left\|u_{h}\right\|_{T}^{2}+h^{-2}\left\|P_{n-2} f\right\|_{T}^{2}\right\}
\end{aligned}
$$

where $\sigma$ is defined by

$$
1+\sigma=\frac{\left(\alpha \cdot n_{1}\right)\left(\alpha \cdot n_{2}\right)}{\left(\alpha(Q) \cdot n_{1}\right)\left(\alpha(Q) \cdot n_{2}\right)}
$$

and

$$
\omega=-\frac{\left(\tau_{1} \cdot n_{3}\right)\left(\tau_{2} \cdot n_{3}\right)}{\left(\alpha(Q) \cdot n_{1}\right)\left(\alpha(Q) \cdot n_{2}\right)} \frac{1}{\alpha \cdot n_{3}} .
$$

Note that $\sigma$ is of order $h$. The terms on the left side of (22) which have $\sigma$ as a factor can therefore be bounded by $C\left\{\left\|\nabla u_{h}\right\|_{T}^{2}+\left\|u_{h}\right\|_{T}^{2}\right\}$. We conclude that (22) remains valid with $\sigma$ deleted.

Note also that $|\omega|$ is bounded away from both 0 (by $\mathrm{H} 1$ ) and $\infty$ (by $\mathrm{H} 2$ ). Moreover, since $\tau_{1} \cdot n_{3}<0$ and $\tau_{2} \cdot n_{3}>0, \omega$ will have the same sign as $\alpha \cdot n_{3}$, which is negative for type I triangles and positive for type II triangles. Thus there exist constants $\mu, \lambda$ such that

(a) $|\omega|<\mu<\infty$ for a triangle of either type,

(b) $\omega>\lambda>0$ for a type II triangle.

This allows us to bound the term

$$
\gamma \equiv \int_{\Gamma_{3}} \omega\left\{\left(u_{h}\right)_{\alpha}^{2}+2 \beta u_{h}\left(u_{h}\right)_{\alpha}\right\} d \tau
$$

in (22) from above for a type I triangle and from below for a type II triangle. 
First consider the case of a type I triangle. Here

$$
|\gamma| \leqq C\left\{\left|\left(u_{h}\right)_{\alpha}\right|_{\Gamma_{3}}^{2}+\left|u_{h}\right|_{\Gamma_{3}}^{2}\right\}
$$

But

$$
\begin{aligned}
\left|\left(u_{h}\right)_{\alpha}\right|_{\Gamma_{3}} & \leqq\left|\left(u_{h}\right)_{\alpha_{0}}\right|_{\Gamma_{3}}+\left|\left(\alpha-\alpha_{0}\right) \cdot \nabla u_{h}\right|_{\Gamma_{3}} \\
& \leqq C\left\{h^{-1 / 2}\left\|\left(u_{h}\right)_{\alpha_{0}}\right\|_{T}+h^{1 / 2}\left\|\nabla u_{h}\right\|_{T}\right\} .
\end{aligned}
$$

Now from (3) we have

$$
\left(u_{h}\right)_{\alpha_{0}}=P_{n-1}\left\{f-\beta u_{h}-\left(\alpha-\alpha_{0}\right) \cdot \nabla u_{h}\right\} .
$$

Hence

$$
\left\|\left(u_{h}\right)_{\alpha_{0}}\right\|_{T} \leqq C\left\{\|f\|_{T}+\left\|u_{h}\right\|_{T}+h\left\|\nabla u_{h}\right\|_{T}\right\} .
$$

Thus we obtain

$$
|\gamma| \leqq C\left\{h^{-1}\|f\|_{T}^{2}+h^{-1}\left\|u_{h}\right\|_{T}^{2}+h\left\|\nabla u_{h}\right\|_{T}^{2}\right\} .
$$

We use (24) in (22), multiply the resulting inequality by $h^{3 / 2}$, then add (17). Application of the estimates (9) and (10) then leads directly to the desired stability result for type I triangles.

Next consider the case of a type II triangle. Here

$$
\begin{gathered}
\gamma>\lambda\left|\left(u_{h}\right)_{\alpha}\right|_{\Gamma_{3}}^{2}-2\|\beta\|_{\infty}\left|u_{h}\right|_{\Gamma_{3}}\left|\left(u_{h}\right)_{\alpha}\right|_{\Gamma_{3}} \\
\geqq(\lambda-\delta)\left|\left(u_{h}\right)_{\alpha}\right|_{\Gamma_{3}}^{2}-\frac{\left(\|\beta\|_{\infty}\left|u_{h}\right|_{\Gamma_{3}}\right)^{2}}{\delta}
\end{gathered}
$$

for any $\delta>0$. We use (25) in (22), multiply the resulting inequality by $h^{3 / 2}$, then add (18). We choose $\delta=\varepsilon=\lambda / 4$ and apply (9) and (10) again. The desired stability result for type II triangles then follows upon setting $\Lambda=\lambda / 2$.

The local results of Theorem 5.2 lead to global stability for $u_{h}$ and $d u_{h} / d \tau$ along interelement boundaries. Before showing this, however, we reformulate Theorem 5.2 in a way that will enable us to control the growth of $\left(u_{h}\right)_{\alpha}$ as well.

THEOREM 5.2a. There exists a positive constant $M$ such that for a triangle $T$ of either type

$$
\begin{gathered}
\int_{\Gamma(T)}\left[\frac{h^{3 / 2}\left(d u_{h} / d \tau\right)^{2}}{\alpha \cdot n}+u_{h}^{2} \alpha \cdot n\right] d \tau-2 h^{3 / 2} \int_{\Gamma(T)} \beta \cot \theta u_{h}\left(u_{h}\right)_{\tau} d \tau+M h^{1 / 2}\left\|\left(u_{h}\right)_{\alpha}\right\|_{T}^{2} \\
\leqq C\left\{h^{1 / 2}\|f\|_{T}^{2}+h^{-1 / 2}\left\|P_{n-2} f\right\|_{T}^{2}+h \int_{\Gamma_{i n}(T)}\left[h^{3 / 2}\left(u_{h}\right)_{\tau}^{2}+u_{h}^{2}\right] d \tau\right\} .
\end{gathered}
$$

Proof. For a type I triangle $T$, we write (3) as

$$
\left(\left(u_{h}\right)_{\alpha_{0}}, v_{h}\right)=\left(f-\beta u_{h}-\left(\alpha-\alpha_{0}\right) \cdot \nabla u_{h}, v_{h}\right) \text { all } v_{h} \in \mathbf{P}_{n-1}(T),
$$

which implies that

$$
\left(u_{h}\right)_{\alpha_{0}}=P_{n-1}\left\{f-\beta u_{h}-\left(\alpha-\alpha_{0}\right) \cdot \nabla u_{h}\right\}
$$

Thus

$$
\left\|\left(u_{h}\right)_{\alpha_{0}}\right\|_{T} \leqq C\left\{\|f\|_{T}+\left\|u_{h}\right\|_{T}+h\left\|\nabla u_{h}\right\|_{T}\right\}
$$

and

$$
\begin{aligned}
\left\|\left(u_{h}\right)_{\alpha}\right\|_{T} & \leqq\left\|\left(u_{h}\right)_{\alpha_{0}}\right\|_{T}+\left\|\left(\alpha-\alpha_{0}\right) \cdot \nabla u_{h}\right\|_{T} \\
& \leqq C\left\{\|f\|_{T}+\left\|u_{h}\right\|_{T}+h\left\|\nabla u_{h}\right\|_{T}\right\} .
\end{aligned}
$$


Squaring and applying Lemma 3.2, we obtain

$$
\left\|\left(u_{h}\right)_{\alpha}\right\|_{T}^{2} \leqq C\left\{\|f\|_{T}^{2}+h\left|u_{h}\right|_{\Gamma_{i n}(T)}^{2}+h^{3}\left|\left(u_{h}\right)_{\tau}\right|_{\Gamma_{i n}(T)}^{2}\right\} .
$$

For any $M>0$, we may add $M h^{1 / 2}$ times the preceding inequality to part (i) of Theorem 5.2 and obtain the desired result for type I triangles.

For a type II triangle $T$, we obtain from Lemma 3.3

$$
\left\|\left(u_{h}\right)_{\alpha}\right\|_{T}^{2} \leqq C\left\{h\left|\left(u_{h}\right)_{\alpha}\right|_{\Gamma_{o u t}(T)}^{2}+\left\|P_{n-2}\left(u_{h}\right)_{\alpha}\right\|_{T}^{2}+h^{2}\left\|\nabla u_{h}\right\|_{T}^{2}\right\} .
$$

Analogous to what has just been done for type I triangles, we find that

$$
P_{n-2}\left(u_{h}\right)_{\alpha}=P_{n-2}\left\{f-\beta u_{h}-\left(\alpha-\alpha_{0}\right) \cdot \nabla u_{h}\right\}
$$

and that

$$
\left\|P_{n-2}\left(u_{h}\right)_{\alpha}\right\|_{T}^{2} \leqq C\left\{\|f\|_{T}^{2}+h\left|u_{h}\right|_{\Gamma_{i n}(T)}^{2}+h^{3}\left|\left(u_{h}\right)_{\tau}\right|_{\Gamma_{i n}(T)}^{2}\right\} .
$$

Using this inequality in (26) and applying Lemma 3.2, we obtain

$$
\left\|\left(u_{h}\right)_{\alpha}\right\|_{T}^{2} \leqq C\left\{h\left|\left(u_{h}\right)_{\alpha}\right|_{\Gamma_{\text {out }}(T)}^{2}+\|f\|_{T}^{2}+h\left|u_{h}\right|_{\Gamma_{i n}(T)}^{2}+h^{3}\left|\left(u_{h}\right)_{\tau}\right|_{\Gamma_{i n}(T)}^{2}\right\} .
$$

Adding $M h^{1 / 2}$ times the above inequality to part (ii) of Theorem 5.2, where $M \equiv \Lambda / C$, produces the desired result for type II triangles.

Before extending Theorem 5.2a into a global stability result, we establish the following simple lemma.

LEMMA 5.3. If

$$
x_{i}+a_{i} \leqq(1+\kappa h) x_{i-1}+b_{i}, \quad i=1,2, \cdots
$$

where $\kappa>0, h>0$ and $x_{i}, a_{i}$, and $b_{i}$ are nonnegative for all $i$, then

$$
x_{n}+\sum_{i=1}^{n} a_{i} \leqq e^{\kappa n h}\left(x_{0}+\sum_{i=1}^{n} b_{i}\right) \text {. }
$$

Proof. The solution of the inequalities (27) is

$$
x_{n} \leqq \sum_{i=1}^{n}(1+\kappa h)^{n-i}\left(b_{i}-a_{i}\right)+(1+\kappa h)^{n} x_{0} .
$$

Thus

$$
x_{n} \leqq(1+\kappa h)^{n}\left(x_{0}+\sum_{i=1}^{n} b_{i}\right)-\sum_{i=1}^{n} a_{i}
$$

The result follows upon noting that

$$
(1+\kappa h)^{n}<e^{\kappa n h}
$$

We are now ready to prove the main stability result of this section.

Proof of Theorem 5.1. For any triangle $T$, we infer from Theorem 5.2a that

$$
\begin{aligned}
\int_{\Gamma_{\text {out }}(T)} & \left.\frac{h^{3 / 2}\left(d u_{h} / d \tau\right)^{2}}{|\alpha \cdot n|}+u_{h}^{2}|\alpha \cdot n|\right\} d \tau \\
& -2 h^{3 / 2} \int_{\Gamma_{\text {out }}(T)} \beta \cot \theta u_{h}\left(u_{h}\right)_{\tau} d \tau+M h^{1 / 2}\left\|\left(u_{h}\right)_{\alpha}\right\|_{T}^{2} \\
\leqq & \int_{\Gamma_{\text {in }}(T)}\left\{\frac{h^{3 / 2}\left(d u_{h} / d \tau\right)^{2}}{|\alpha \cdot n|}+u_{h}^{2}|\alpha \cdot n|\right\} d \tau+2 h^{3 / 2} \int_{\Gamma_{\text {in }}(T)} \beta \cot \theta u_{h}\left(u_{h}\right)_{\tau} d \tau \\
& +C\left\{h^{1 / 2}\|f\|_{T}^{2}+h^{-1 / 2}\left\|P_{n-2} f\right\|_{T}^{2}+h \int_{\Gamma_{\text {in }}(T)}\left[h^{3 / 2}\left(u_{h}\right)_{\tau}^{2}+u_{h}^{2}\right] d \tau\right\} .
\end{aligned}
$$


Summation over all triangles $T \in S_{j}$ yields

$$
p_{j}+q_{j}+M h^{1 / 2}\left\|\left(u_{h}\right)_{\alpha}\right\|_{S_{j}}^{2} \leqq\{1+O(h)\} p_{j-1}+q_{j-1}+C\left\{h^{1 / 2}\|f\|_{S_{j}}^{2}+h^{-1 / 2}\left\|P_{n-2} f\right\|_{S_{j}}^{2}\right\}
$$

where

$$
p_{j} \equiv \int_{F_{j}}\left\{\frac{h^{3 / 2}\left(d u_{h} / d \tau\right)^{2}}{|\alpha \cdot n|}+u_{h}^{2}|\alpha \cdot n|\right\} d \tau
$$

and

$$
q_{j} \equiv 2 h^{3 / 2} \int_{F_{j}} \beta \cot \theta u_{h}\left(u_{h}\right)_{\tau} d \tau,
$$

with the convention that integrals over $F_{j}$ are taken left to right (thus fixing the sign of $\left(u_{h}\right)_{\tau}$ in the definition of $\left.q_{j}\right)$.

Next, we note that

$$
\left|q_{j}\right| \leqq C h^{3 / 4} p_{j}
$$

(an immediate consequence of the Schwarz and arithmetic-geometric mean inequalities). Hence

$$
\left|q_{j}\right| \leqq p_{j}+q_{j}
$$

for $h$ sufficiently small. Thus in (29) we may write

$$
\begin{aligned}
\{1+O(h)\} p_{j-1}+q_{j-1} & =\{1+O(h)\}\left(p_{j-1}+q_{j-1}\right)+O(h) q_{j-1} \\
& \leqq\{1+O(h)\}\left(p_{j-1}+q_{j-1}\right) .
\end{aligned}
$$

Equation (29) then becomes

$$
\begin{aligned}
& p_{j}+q_{j}+M h^{1 / 2}\left\|\left(u_{h}\right)_{\alpha}\right\|_{S_{j}}^{2} \\
& \leqq\{1+O(h)\}\left(p_{j-1}+q_{j-1}\right)+C\left\{h^{1 / 2}\|f\|_{S_{j}}^{2}+h^{-1 / 2}\left\|P_{n-2} f\right\|_{S_{j}}^{2}\right\} .
\end{aligned}
$$

Inequality (31) implies that $p_{j}+q_{j} \geqq 0$ for $h$ sufficiently small, in which case Lemma 5.3 is applicable to (32). Using hypothesis $\mathrm{H} 4$, we get

$$
p_{j}+q_{j}+M h^{1 / 2}\left\|\left(u_{h}\right)_{\alpha}\right\|_{\Omega_{j}}^{2} \leqq C\left\{p_{0}+q_{0}+h^{1 / 2}\|f\|_{\Omega_{j}}^{2}+h^{-1 / 2}\left\|P_{n-2} f\right\|_{\Omega_{j}}^{2}\right\} .
$$

Using (30) again, we note that

$$
p_{j}+q_{j}=p_{j}\left\{1+O\left(h^{3 / 4}\right)\right\},
$$

and infer that (33) remains valid with $q_{j}$ and $q_{0}$ deleted. The result is Theorem 5.1.

As a consequence of Theorem 5.1 and Lemma 3.2, we can now establish stability for $u_{h}, \nabla u_{h}$ and $\left(u_{h}\right)_{\alpha}$ over $\Omega$.

THEOREM 5.3.

$$
\begin{aligned}
\left\|u_{h}\right\|_{\Omega}^{2} & +h^{3 / 2}\left\|\nabla u_{h}\right\|_{\Omega}^{2}+h^{1 / 2}\left\|\left(u_{h}\right)_{\alpha}\right\|_{\Omega}^{2} \\
& \leqq C\left\{h^{1 / 2}\|f\|_{\Omega}^{2}+h^{-1 / 2}\left\|P_{n-2} f\right\|_{\Omega}^{2}+h^{3 / 2}\left|\frac{d u_{h}}{d \tau}\right|_{\Gamma_{i n}(\Omega)}^{2}+\left|u_{h}\right|_{\Gamma_{i n}(\Omega)}^{2}\right\} .
\end{aligned}
$$

Proof. From Lemma 3.2, we have

$$
\left\|u_{h}\right\|_{S_{j}}^{2} \leqq C\left\{h\left|u_{h}\right|_{F_{j-1}}^{2}+h^{2}\|f\|_{S_{j}}^{2}\right\}
$$

and

$$
\left\|\nabla u_{h}\right\|_{S_{j}}^{2} \leqq C\left\{h\left|\frac{d u_{h}}{d \tau}\right|_{F_{j-1}}^{2}+h\left|u_{h}\right|_{F_{j-1}}^{2}+\|f\|_{S_{j}}^{2}\right\} .
$$


Thus

$$
\begin{aligned}
\left\|u_{h}\right\|_{S_{j}}^{2}+h^{3 / 2}\left\|\nabla u_{h}\right\|_{S_{j}}^{2} & \leqq C\left\{h\left|u_{h}\right|_{F_{j-1}}^{2}+h^{5 / 2}\left|\frac{d u_{h}}{d \tau}\right|_{F_{j-1}}^{2}+h^{3 / 2}\|f\|_{S_{j}}^{2}\right\} \\
& \leqq C h \int_{F_{j-1}}\left\{\frac{h^{3 / 2}\left(d u_{h} / d \tau\right)^{2}}{|\alpha \cdot n|}+u_{h}^{2}|\alpha \cdot n|\right\} d \tau+h^{3 / 2}\|f\|_{S_{j}}^{2} .
\end{aligned}
$$

Applying Theorem 5.1, we obtain

$$
\left\|u_{h}\right\|_{S_{j}}^{2}+h^{3 / 2}\left\|\nabla u_{h}\right\|_{S_{j}}^{2} \leqq C h\left\{h^{1 / 2}\|f\|_{\Omega_{j}}^{2}+h^{-1 / 2}\left\|P_{n-2} f\right\|_{\Omega_{j}}^{2}+h^{3 / 2}\left|\frac{d u_{h}}{d \tau}\right|_{\Gamma_{i n}(\Omega)}^{2}+\left|u_{h}\right|_{\Gamma_{i n}(\Omega)}^{2}\right\} .
$$

Summation over all layers $S_{j}$ yields

$$
\begin{aligned}
& \left\|u_{h}\right\|_{\Omega}^{2}+h^{3 / 2}\left\|\nabla u_{h}\right\|_{\Omega}^{2} \\
& \leqq C\left\{h^{1 / 2}\|f\|_{\Omega}^{2}+h^{-1 / 2}\left\|P_{n-2} f\right\|_{\Omega}^{2}+h^{3 / 2}\left|\frac{d u_{h}}{d \tau}\right|_{\Gamma_{i n}(\Omega)}^{2}+\left|u_{h}\right|_{\Gamma_{i n}(\Omega)}^{2}\right\} .
\end{aligned}
$$

To account for $\left\|\left(u_{h}\right)_{\alpha}\right\|_{\Omega}^{2}$, we apply Theorem 5.1 with $F_{j}=\Gamma_{o u t}(\Omega)$ to conclude that

$$
h^{1 / 2}\left\|\left(u_{h}\right)_{\alpha}\right\|_{\Omega}^{2} \leqq C\left\{h^{1 / 2}\|f\|_{\Omega}^{2}+h^{-1 / 2}\left\|P_{n-2} f\right\|_{\Omega}^{2}+h^{3 / 2}\left|\frac{d u_{h}}{d \tau}\right|_{\Gamma_{i n}(\Omega)}^{2}+\left|u_{h}\right|_{\Gamma_{i n}(\Omega)}^{2}\right\} .
$$

The statement in the theorem is the sum of (34) and (35).

6. Error estimates. To obtain error estimates for the method, we define an interpolant $u_{I} \in S_{h}^{n}$ by the following conditions:

(i) $u_{I}\left(a_{i}\right)=u\left(a_{i}\right)$ for all triangle vertices $a_{i}$;

(ii) $\int_{\Gamma}\left(u_{I}-u\right) \tau^{l} d \tau=0, l=0,1, \cdots, n-2$ for all triangle sides $\Gamma$;

(iii) $\int_{T}\left(u_{I}-u\right) q d x d y=0$ for all $q \in \mathbf{P}_{n-3}(T)$ and all triangles $T$.

It is straightforward to show (for example, using the techniques in [3, Chap. 3]) that $u_{I}$ has the following approximation properties:

$$
\left\|u-u_{I}\right\|_{j, T} \leqq C h^{n+1-j}\|u\|_{n+1, T}, \quad j=0,1
$$

and

$$
\left|u-u_{I}\right|_{j, \Gamma(T)} \leqq C h^{n+1 / 2-j}\|u\|_{n+1, T}, \quad j=0,1 .
$$

Rewriting (3) and (4) in the form

$$
\left(\left(u_{h}-u_{I}\right)_{\alpha}+\beta\left(u_{h}-u_{I}\right), v_{h}\right)_{T}=\left(\left(u-u_{I}\right)_{\alpha}+\beta\left(u-u_{I}\right), v_{h}\right)_{T},
$$

we may apply Theorems 5.1 and 5.3 with $u_{h}$ replaced by $u_{h}-u_{I}$, and $f$ replaced by $r \equiv\left(u-u_{I}\right)_{\alpha}+\beta\left(u-u_{I}\right)$.

From (36), it follows immediately that

$$
\|r\|_{\Omega_{j}} \leqq C h^{n}\|u\|_{n+1, \Omega_{j}} .
$$

Moreover, for all $v_{h} \in \mathbf{P}_{n-2}(T)$,

$$
\left(\left(u-u_{I}\right)_{\alpha_{0}}, v_{h}\right)_{T}=\int_{\Gamma}\left(u-u_{I}\right) v_{h} \alpha_{0} \cdot n d \Gamma-\left(\left(u-u_{I}\right),\left(v_{h}\right)_{\alpha_{0}}\right)_{T}=0
$$

since $\left(v_{h}\right)_{\alpha_{0}} \in \mathbf{P}_{n-3}(T)$. Hence

$$
\begin{aligned}
\left\|P_{n-2} r\right\|_{\Omega_{j}} & \leqq\left\|P_{n-2}\left(\alpha-\alpha_{0}\right) \cdot \nabla\left(u-u_{I}\right)\right\|_{\Omega_{j}}+\left\|\beta\left(u-u_{I}\right)\right\|_{\Omega_{j}} \\
& \leqq C h^{n+1}\|u\|_{n+1, \Omega_{j}} .
\end{aligned}
$$


We also assume, for convenience, that $u_{h}=u_{I}$ on $\Gamma_{i n}(\Omega)$. Insertion of these bounds into Theorems 5.1 and 5.3 now yields the following theorem.

THEOREM 6.1. Let $u$ be the solution of (1) and $u_{h}$ the solution of Problem $\mathbf{P}_{\mathrm{h}}$. If $u \in H^{n+1}(\Omega)$, there exists a constant $C$ independent of $h$ such that

$$
\begin{aligned}
& \left\|u-u_{h}\right\|_{\Omega} \leqq C h^{n+1 / 4}\|u\|_{n+1, \Omega}, \\
& \left\|\nabla\left(u-u_{h}\right)\right\|_{\Omega} \leqq C h^{n-1 / 2}\|u\|_{n+1, \Omega}, \\
& \left\|\left(u-u_{h}\right)_{\alpha}\right\| \leqq C h^{n}\|u\|_{n+1, \Omega},
\end{aligned}
$$

and for $j=1,2, \cdots$

and

$$
\left\{\int_{F_{j}}\left(u-u_{h}\right)^{2} d \tau\right\}^{1 / 2} \leqq C h^{n+1 / 4}\|u\|_{n+1, \Omega_{j}}
$$

$$
\left\{\int_{F_{j}}\left[\frac{d}{d \tau}\left(u-u_{h}\right)\right]^{2} d \tau\right\}^{1 / 2} \leqq C h^{n-1 / 2}\|u\|_{n+1, \Omega_{j}}
$$

7. Computational results. We shall present numerical results for three test problems, each involving approximation by piecewise quadratics. The first problem is

$$
.6 u_{x}+.8 u_{y}-u=0, \quad 0<x<1, \quad 0<y<1 \text {, }
$$

with initial data along the $x$ and $y$ axes chosen to make the solution be $u=$ $\exp (.6 x+.8 y)$. A triangulation was obtained by dividing the domain uniformly into $N^{2}$ squares, then dividing each square into two right triangles by drawing a diagonal parallel to the vector $\left(\begin{array}{c}-1 \\ 1\end{array}\right)$. In Table 6.1, values are presented for the quantities

$$
\begin{aligned}
& E \equiv \sqrt{\int_{\Gamma_{o u t}(\Omega)}\left(u_{h}-u\right)^{2} \alpha \cdot n d \tau}, \\
& E^{\prime} \equiv \sqrt{\int_{\Gamma_{\text {out }}(\Omega)} \frac{\left(d u_{h} / d \tau\right)^{2}}{\alpha \cdot n} d \tau}
\end{aligned}
$$

and ratios of consecutive values of these quantities as $N$ is doubled.

Our theory predicts errors of order $h^{n+1 / 4}$ in $u_{h}$ and $h^{n-1 / 2}$ in $d u_{h} / d \tau$. If these estimates correctly described the rates of convergence of $u_{h}$ and $d u_{h} / d \tau$, the ratios of consecutive errors in these quantities would have limiting values $2^{2.25}=4.76$ and $2^{1.5}=2.83$, respectively. In fact, the ratios in Table 6.1 are consistently larger than these values, indicating a convergence rate similar to that of an interpolant of the exact solution. This example illustrates the tendency of the method to achieve the optimal

TABLE 6.1

Numerical results for first test problem.

\begin{tabular}{rcccc}
\hline$N$ & $E$ & Ratio & $E^{\prime}$ & Ratio \\
\hline 2 & $.735(-3)$ & - & $.132(-1)$ & - \\
4 & $.985(-4)$ & 7.46 & $.355(-2)$ & 3.73 \\
8 & $.136(-4)$ & 7.23 & $.917(-3)$ & 3.87 \\
16 & $.182(-5)$ & 7.48 & $.233(-3)$ & 3.94 \\
32 & $.235(-6)$ & 7.37 & $.587(-4)$ & 3.97 \\
64 & $.299(-7)$ & 7.86 & $.147(-4)$ & 3.98 \\
128 & $.379(-8)$ & 7.93 & $.369(-5)$ & 3.99 \\
\hline
\end{tabular}


order of approximation on problems with a very smooth $u$ and a regular triangulation. A partial explanation may be found in [10].

The second test problem is

$$
u_{x}+u_{y}=0, \quad 0<x<1, \quad 0<y<1
$$

with initial data chosen to make the solution be $u=|t|^{2.5+\varepsilon} / 2.5, \varepsilon \ll 1$, where $t=$ $(y-x) / \sqrt{2}$ is a coordinate orthogonal to the characteristic direction. The domain and triangulation are as in the previous problem. Here there is no extra differentiability in $u$ beyond what is needed for applicability of our theoretical estimates. In this case, the ratios appear to be consistent with the theoretical predictions (see Table 6.2).

The last test problem is

$$
\sqrt{3} u_{t}+u_{x}=0, \quad-\infty<x<\infty, \quad t>0,
$$

with a discontinuous initial condition:

$$
u(x, 0)= \begin{cases}1, & x<0 \\ .5, & x=0 \\ 0, & x>0\end{cases}
$$

The discontinuity propagates away from $(x, t)=(0,0)$ along a line making an angle $60^{\circ}$ with the $x$-axis. We again use a uniform set of right isosceles triangles in the triangulation, but here the hypotenuses are taken parallel to the $x$-axis.

Both the continuous and discontinuous finite element methods were applied to this problem. Table 6.3 indicates the $L^{2}$ error in $u_{h}$ at $t=1$ and $t=2$, displayed as functions of the hypotenuse length $h$ (the time step is $\Delta t=h / 2$ ). For the range of

TABLE 6.2

Numerical results for second test problem.

\begin{tabular}{rcccc}
\hline$N$ & $E$ & Ratio & $E^{\prime}$ & Ratio \\
\hline 2 & $.509(-2)$ & - & $.412(-1)$ & - \\
4 & $.785(-3)$ & 6.49 & $.110(-1)$ & 3.73 \\
8 & $.141(-3)$ & 5.58 & $.312(-2)$ & 3.53 \\
16 & $.286(-4)$ & 4.93 & $.977(-3)$ & 3.20 \\
32 & $.598(-5)$ & 4.78 & $.297(-3)$ & 3.29 \\
64 & $.129(-5)$ & 4.65 & $.100(-3)$ & 2.97 \\
128 & $.276(-6)$ & 4.66 & $.338(-4)$ & 2.96 \\
\hline
\end{tabular}

TABLE 6.3

$L^{2}$ errors for third test problem.

\begin{tabular}{rlllc}
\hline \multicolumn{1}{c}{$h$} & \multicolumn{2}{c}{ Continuous method } & \multicolumn{2}{c}{ Discontinuous method } \\
$t=1$ & $t=1$ & $t=2$ & $t=2$ \\
\hline 1 & .298 & .377 & .247 & .231 \\
.5 & .267 & .263 & .163 & .182 \\
.25 & .186 & .194 & .129 & .137 \\
.125 & .137 & .157 & .0970 & .103 \\
.0625 & .111 & .117 & .0725 & .0784 \\
.03125 & .0830 & .0894 & .0554 & .0577 \\
.015625 & .0632 & .0680 & .0408 & .0434 \\
.0078125 & .0481 & .0519 & .0307 & .0327 \\
\hline
\end{tabular}


discretizations presented, the discontinuous method yields an error $29-39 \%$ less than the continuous method. However, the discontinuous piecewise quadratic has three times as many degrees of freedom over the triangulation as does its continuous counterpart. Provision for computational cost would thus reduce the advantage of the discontinuous method on this problem.

Finally, in Figs. 6.1 and 6.2, the continuous and discontinuous solutions are displayed for $h=.03125$ at time $t=2$ (128 time steps). The discontinuity has been smoothed more by the continuous method than by the discontinuous method.

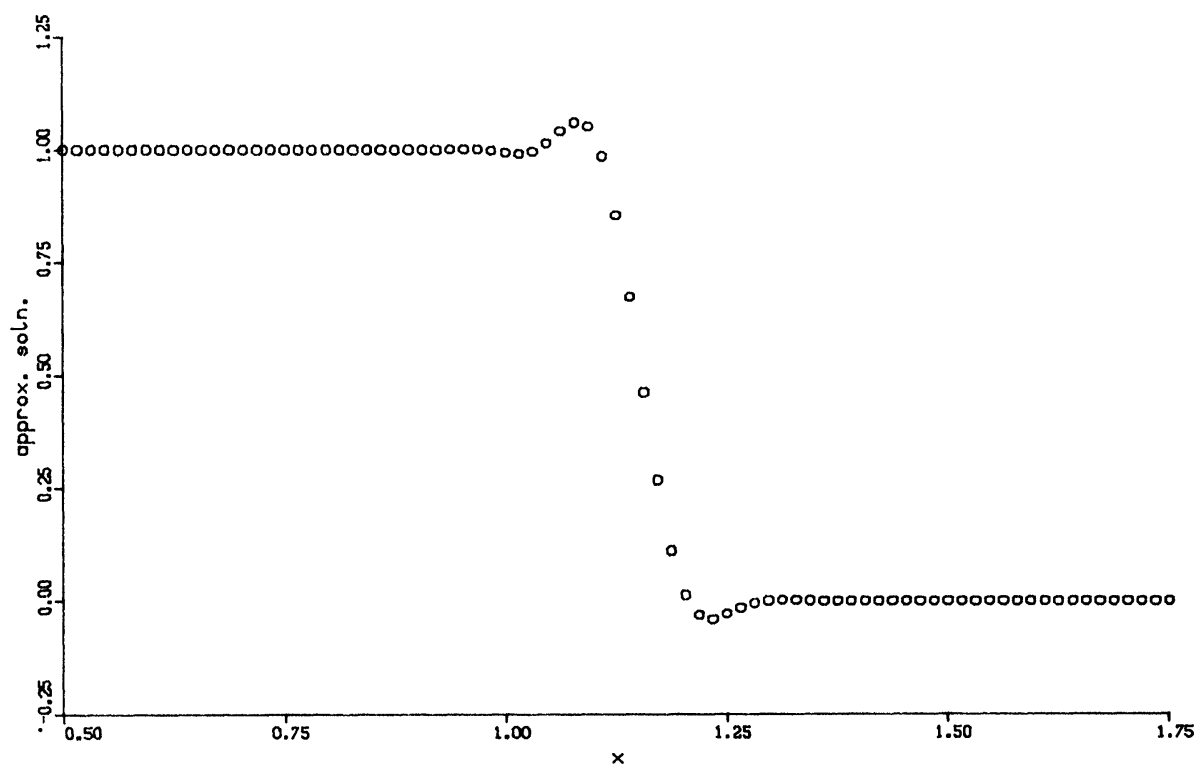

FIG. 6.1. Continuous method: $t=2$.

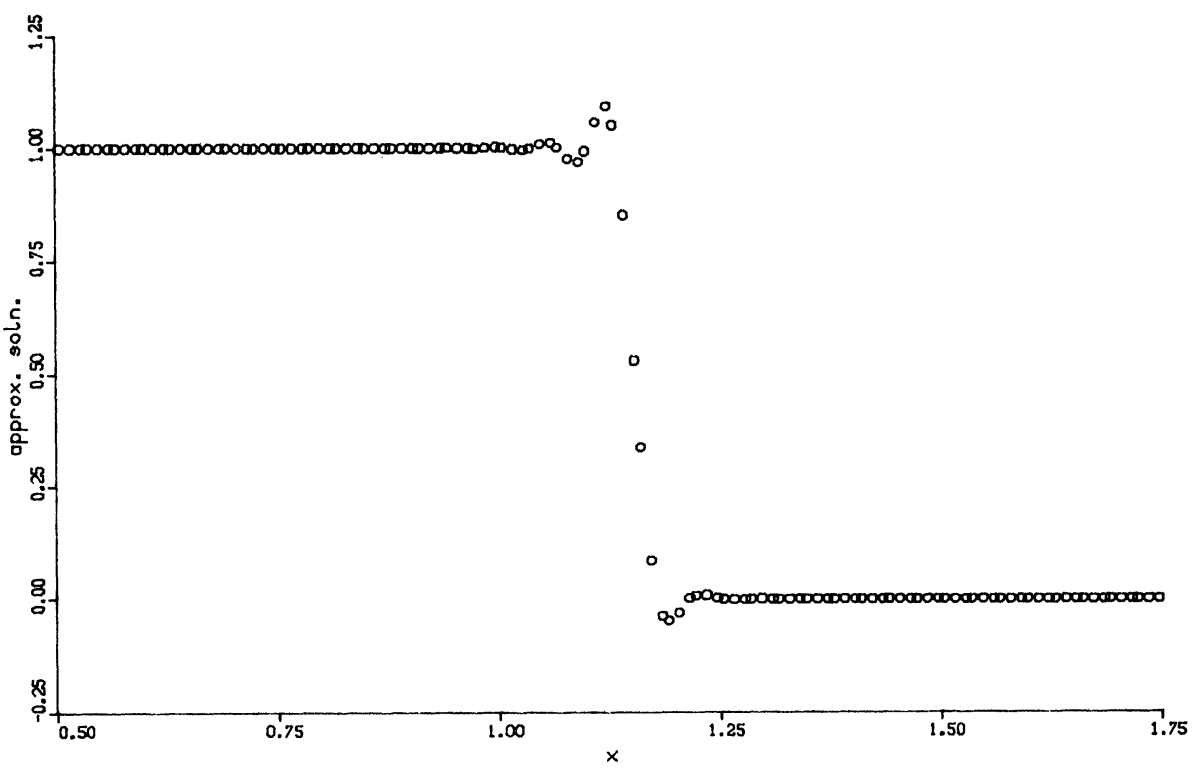

FIG. 6.2. Discontinuous method: $t=2$. 
Appendix. Suppose $\Delta_{h}=\left\{T_{1}, T_{2}, \cdots, T_{N}\right\}$ is a triangulation of $\Omega$ such that $|\alpha \cdot n|>0$ along every side of every triangle, where $\alpha$ is continuous and of unit length over $\Omega$. We will show that the triangles in $\Delta_{h}$ can be ordered such that

$$
\Gamma_{\text {in }}\left(T_{k}\right) \subseteq \Gamma_{\text {out }}\left(\bigcup_{i<k} T_{i}\right) \cup\left(\Gamma_{\text {in }}(\Omega)-\Gamma_{\text {in }}\left(\bigcup_{i<k} T_{i}\right)\right) \text {. }
$$
triangles

Definition A1. A path from $T_{j} \in \Delta_{h}$ to $T_{k} \in \Delta_{h}$ is an ordered set of 2 or more

$$
T_{i_{1}}, \cdots, T_{i_{m}}
$$

such that $T_{i_{1}}=T_{j}, T_{i_{m}}=T_{k}$ and for $l=1, \cdots, m-1, T_{i_{l}}$ and $T_{i_{l+1}}$ have a common side which serves as outflow from $T_{i_{l}}$ and inflow to $T_{i_{l+1}}$.

Definition A2. A cycle is a path from a triangle to itself.

LEMMA A.1.

(i) $\Delta_{h}$ has no cycles;

(ii) There exists a triangle whose inflow is a subset of $\Gamma_{i n}(\Omega)$;

(iii) The triangles in $\Delta_{h}$ can be ordered in a manner consistent with (38).

Proof. (i). Suppose there were a cycle with enclosed triangles as depicted in Fig. A.1, and consider any characteristic $\mathbf{C}$ inside the enclosed region. $\mathbf{C}$ must enter the enclosed region from some triangle $A$ of the cycle, and leave it via another triangle $B$ of the cycle. Moreover, the set of triangles intersecting with this portion of $\mathbf{C}$ forms a path through the interior from $A$ to $B$. This path permits formation of a new cycle having fewer enclosed triangles than the original one. Continuing in this manner, we eventually obtain a cycle with no enclosed triangles, as shown in Fig. A.2. However, Fig. A.2 describes a situation where, at the common vertex $P$, the value of $\alpha$ is such that $\alpha(P) \cdot n_{i}$ has the same sign for each of the normals $n_{i}$ depicted in the figure. But $\alpha$, by assumption, is well defined at $P$, so $\alpha(P) \cdot x$ will be of constant sign only for vectors $x$ lying in some half plane. It is geometrically impossible for all the normals $n_{i}$ to lie in a common half plane. Thus there cannot be any cycles.

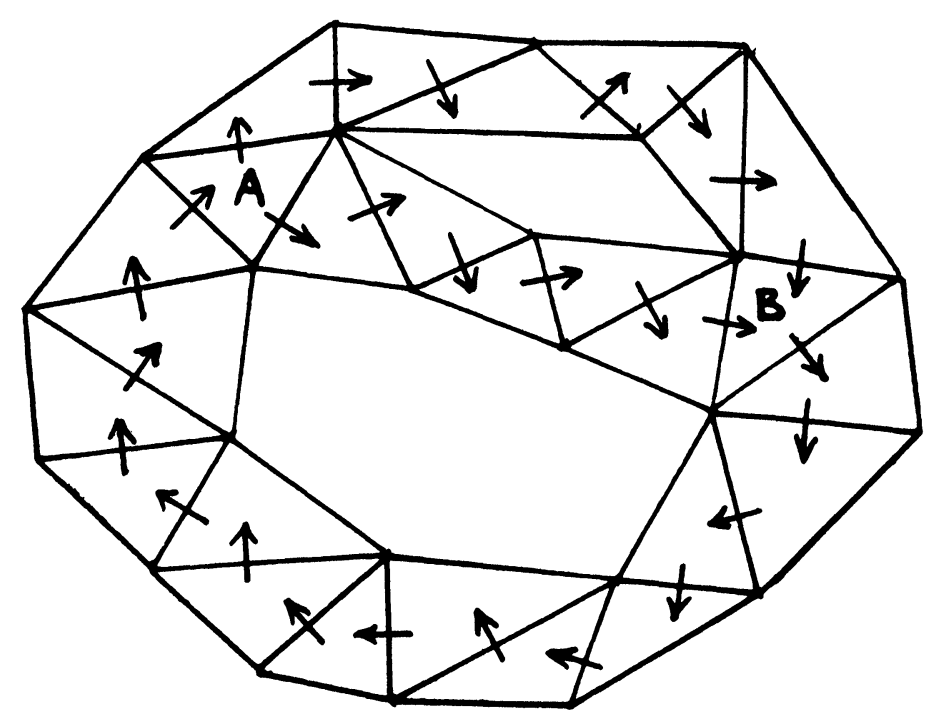

FIG. A.1 


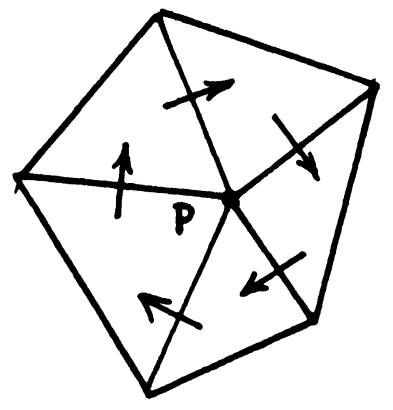

Fig. A.2

(ii) If the inflow to $T_{i}$ is not a subset of $\Gamma_{i n}(\Omega)$, there must be a path to $T_{i}$ from another triangle $T_{j}, j \neq i$. If this were true for all triangles $T_{i} \in \Delta_{h}$, there would have to be a cycle, which was ruled out in part (i) of the lemma.

(iii) This follows by induction, using part (ii) of the lemma.

\section{REFERENCES}

[1] G. A. BAKER, A finite element method for first order hyperbolic equations, Math. Comp., 29 (1975), pp. 995-1006.

[2] J. BEAR, Dynamics of Fluids in Porous Media, American Elsevier, New York, 1972, pp. 214-215.

[3] P. G. CiARLet, The Finite Element Method for Elliptic Problems, North-Holland, Amsterdam, 1978.

[4] T. DUPONT, Galerkin methods for modelling gas pipelines, in Constructive and Computational Methods for Differential and Integral Equations, Lecture Notes in Mathematics, Vol. 430, Springer-Verlag, Berlin-Heidelberg-New York, 1974.

[5] R. S. FALK, Error estimates for the numerical identification of a variable coefficient, Math. Comp., 40 (1983), pp. 537-546.

[6] C. Johnson AND J. Pitkaranta, An analysis of the discontinuous Galerkin method for a scalar hyperbolic equation, Math. Comp., 46 (1986), pp. 1-26.

[7] P. LESAINT AND P. A. RAVIART, On a finite element method for solving the neutron transport equation, in Mathematical Aspects of Finite Elements in Partial Differential Equations, C. de Boor, ed., Academic Press, New York, 1974, pp. 89-123.

[8] W. H. REED AND T. R. HILl, Triangular mesh methods for the neutron transport equation, Los Alamos Scientific Laboratory Report LA-UR-73-479, 1973.

[9] G. R. RICHTER, An inverse problem for the steady state diffusion equation, SIAM J. Appl. Math., 41 (1981), pp. 210-221.

[10] — A Ainite element method for hyperbolic equations, Dept. of Computer Science Technical Report No. 138, Rutgers Univ., New Brunswick, NJ, 1983.

[11] R. Winther, A stable finite element method for first-order hyperbolic systems, Math. Comp., 36 (1981), pp. 65-86. 